\title{
Screened fifth forces mediated by dark matter-baryon interactions: Theory and astrophysical probes
}

\author{
Jeremy Sakstein $\odot,{ }^{1, *}$ Harry Desmond, ${ }^{2, \dagger}$ and Bhuvnesh Jain ${ }^{1, *}$ \\ ${ }^{1}$ Center for Particle Cosmology, Department of Physics and Astronomy, University of Pennsylvania, \\ 209 South 33rd Street, Philadelphia, Pennsylvania 19104, USA \\ ${ }^{2}$ Astrophysics, University of Oxford, Denys Wilkinson Building, Keble Road, \\ Oxford OX1 3RH, United Kingdom
}

(Received 15 July 2019; published 18 November 2019)

\begin{abstract}
We derive the details of a new screening mechanism where the interactions of baryons and dark matter can be screened according to the local dark matter density. In this mechanism, the value of Newton's constant is dark matter-density dependent, allowing for the possibility that astrophysical phenomena are very different in galaxies less dense than the Milky Way. The parametrized post-Newtonian parameter $\gamma$, which quantifies the difference between kinematical and lensing probes, also depends on dark matter density. We calculate the effects of varying $G$ on various stages of stellar evolution, focusing on observables that impact cosmology: the Cepheid period-luminosity relation and the supernova Ia magnitude-redshift relation. Other potential tests of the model are also investigated including main-sequence, post-main-sequence, and low-mass dwarf stars. Finally, we discuss how extragalactic tests of $\gamma$ could provide complementary constraints.
\end{abstract}

DOI: 10.1103/PhysRevD.100.104035

\section{INTRODUCTION}

Screening mechanisms play a paramount role in modern cosmology. Using nonlinear effects, they act to suppress potential deviations from the standard $\Lambda$ cold dark matter $(\Lambda \mathrm{CDM})$ cosmological model on small scales by hiding the new or fifth forces that are ubiquitous in theories of dark energy that include new degrees of freedom (d.o.f.) (typically a light scalar) coupled to matter [1-8]. Typically, they are efficient in high-density regions such as the Solar System and the Milky Way but do not operate at low densities in order to allow for novel cosmological phenomenologies. Models of dark energy that do not include screening mechanisms are typically fine tuned or ruled out (see Refs. $[9,10]$ for some exceptions). This has made screening mechanisms the focus of much theoretical study [2], the inspiration for several laboratory tests $[5,6]$ specifically designed to search for them, and targets for upcoming cosmological surveys.

The most commonly studied mechanisms focus on screening interactions between a hypothetical new scalar and matter, although new vectors can also have screening mechanisms [11], as can theories where the graviton is massive [12]. Focusing on scalars, ${ }^{1}$ present screening

\footnotetext{
*sakstein@physics.upenn.edu

tharry.desmond@physics.ox.ac.uk

tbjain@physics.upenn.edu

${ }^{1}$ The screening mechanisms in vector and massive gravity theories can be phrased in terms of equivalent scalar screening mechanisms either by using the Stückelberg trick or by considering the decoupling limit.
}

mechanisms fall into one of two categories. The chameleon $[13,14]$, symmetron [15], and dilaton [16] models screen by suppressing the new scalar charge, which quantifies an object's response to a fifth force field. Conversely, Vainshtein $[12,17]$ and K-mouflage [18] screening suppresses the fifth force field compared with the Newtonian one.

Any new screening mechanism would have profound consequences for cosmology and gravitation because it would allow for the construction of novel nontrivial dark energy models and modified gravity theories. A new screening mechanism is apposite now more than ever, since the binary neutron star merger with optical counterpart has ruled out a plethora of dark energy models that utilize those mechanisms [19-22]. Others, in particular chameleon, symmetron, and dilaton models, are already constrained to levels where they cannot explain dark energy [23].

It is widely believed that no new screening mechanism exists, based on the following argument. Consider a scalar $\phi$ coupled to matter represented by an energy-momentum tensor $T_{\mu \nu}$, and perturb both about their background values so that $\phi=\bar{\phi}+\delta \phi, T_{\mu \nu}=\bar{T}_{\mu \nu}+\delta T_{\mu \nu}$. The most general effective action for the perturbations is (ignoring disformal couplings, which do not give rise to new screening mechanisms $[24,25])$,

$$
\delta S=\int \mathrm{d}^{4} x\left[Z^{\mu \nu}(\bar{\phi}) \partial_{\mu} \delta \phi \partial_{\nu} \delta \phi-m_{\mathrm{eff}}(\bar{\phi}) \delta \phi^{2}+\beta(\bar{\phi}) \frac{\delta \phi}{M_{\mathrm{pl}}} \delta T\right]
$$


where $\delta T=\eta^{\mu \nu} \delta T_{\mu \nu}$ is the trace of the perturbed energymomentum tensor. This includes a noncanonical kinetic term $Z^{\mu \nu}$, an effective mass $m_{\mathrm{eff}}(\bar{\phi})$, and a coupling to matter $\beta(\bar{\phi})$, all of which are background dependent. This action gives rise to a scalar-mediated fifth force

$$
F_{5} \sim \frac{2 \beta^{2}(\bar{\phi}) G M}{\sqrt{Z} r^{2}} e^{-m_{\mathrm{eff}}(\bar{\phi}) r},
$$

where $Z \sim \operatorname{det}\left(Z^{\mu \nu}\right)$. Upon inspection, one can discern three possible methods of suppressing this environmentally. One possibility is that the Compton wavelength $\lambda_{\mathrm{C}} \sim m_{\mathrm{eff}}(\bar{\phi})^{-1}$ is smaller than a micron so that current laboratory tests are satisfied [26-28]. This is how the chameleon mechanism operates. Another is that the coupling to matter is small enough to evade fifth force searches in the Solar System [29]. This is how the symmetron and dilaton mechanisms screen. The final is to have a large kinetic matrix $Z$, as is the case with the Vainshtein and K-mouflage mechanisms. The argument that there are no new screening mechanisms is that any new scalar interactions (either self or with matter) will ultimately be encapsulated in Eq. (2).

Recently, the authors of Ref. [30] have devised a dark energy model where the cosmic acceleration is driven not by new dynamical d.o.f. but by dark matter-baryon interactions. In particular, the Jordan frame metric, $\tilde{g}_{\mu \nu}$, which baryons couple to, is a combination of the Einstein frame metric, $g_{\mu \nu}$, which couples to dark matter, and the dark matter four-velocity $u_{\mu}$ :

$$
\tilde{g}_{\mu \nu}=R^{2}\left(\rho_{\mathrm{DM}}\right)\left(g_{\mu \nu}+u_{\mu} u_{\nu}\right)-Q^{2}\left(\rho_{\mathrm{DM}}\right) u_{\mu} u_{\nu} .
$$

Here, $R$ and $Q$ are arbitrary functions that are assumed to tend to unity (so that $\tilde{g}_{\mu \nu}=g_{\mu \nu}$ ) at early times, or, equivalently, when the dark matter density is high, but become important around the present epoch and drive the cosmic acceleration without any need for dark energy. The authors speculated that any deviations from general relativity (GR) in high dark matter-density environments would be highly suppressed as a necessary corollary of $R$ and $Q$ tending to unity at early times/high densities. This circumvents the argument that there cannot be new screening mechanisms because there are no new d.o.f. in this model, only the standard model, GR, and dark matter.

The reader may recognize the coupling of Eq. (3) as a disformal transformation of the metric. It is well known that disformal transformations of this type can give rise to higher-derivative and nonlinear derivative interactionsHorndeski and its extensions-when one inverts the transformation to work in the Jordan frame [31-34], so the reader may wonder if our mechanism is one of the known mechanisms masquerading in the Einstein frame. This is not the case. One can see this by noting that Horndeski (and its extensions) only admit an Einstein frame if the effects of the nonlinearities can be removed by a disformal transformation [31]. If one were to construct the Jordan frame action, then all of the nonlinear terms would cancel in the equations of motion. Another way to see that our mechanism is unique is to note that our derivation is linear in the new field-by which we mean that the screening does not rely on the solution to a nonlinear equation for the dark matter field sourced by an object-whereas other screening mechanisms rely crucially on nonlinearities to operate.

In this work, we study this theory in detail and verify that a novel screening mechanism indeed exists. In particular, we will show that, as a consequence of the interactions:

C.1 Newton's constant becomes dark matter-density dependent, i.e., $G=G\left(\rho_{\mathrm{DM}}\right)$.

C.2 The parametrized post-Newtonian (PPN) parameter $\gamma$ becomes dark matter-density dependent.

Regardless of dark energy-one can always consider theories with screened fifth forces that are not cosmologically important, as is the case with a large portion of the chameleon and symmetron parameter space as well as the quartic and quintic Galileons and generalizations thereofa basic requirement for any theory producing C. 1 and C. 2 is that the Solar System is screened; otherwise, stringent tests of gravity already performed would be violated [29,35]. It is likely that a vast portion of the Milky Way (MW) is also screened since several recent tests of gravity aimed at constraining fifth forces apply here [6,7]. This implies that tests of the theory should focus on extragalactic observables, including galaxies in halos less dense than that of the MW, and voids (including the local void). To this end, in Secs. IV-VII, we will study the consequences of C.1 for astrophysical objects that can be observed in other galaxies, with the ultimate aim of identifying novel astrophysical probes of this theory. It is possible that some objects could be unscreened in our own Galaxy in regions where the dark matter density is smaller than in the solar neighborhood. For this reason, we also discuss potential probes within the MW in Sec. VIII. Astrophysical tests have been incredibly successful for constraining other screening mechanisms [29,36-50]. In Sec. IX, we discuss how C.2 could be used to further constrain the theory using extragalactic tests comparing lensing and kinematical tracers of the gravitational potential.

The layout of the paper is as follows. In Sec. II, we review the model of Ref. [30] and discuss its salient features. In Sec. III, we derive the new screening mechanism, in particular C.1 and C.2, and discuss possible tests. The main astrophysical test we will consider is devised in Sec. IV. Here, we study the implications of C.1 for post-main-sequence stars with masses in the range $5 M_{\odot}-13 M_{\odot}$. Stars with these masses may undergo Cepheid pulsations, and we derive the change in the period-luminosity relation (PLR), which is used to measure the distance to extragalactic hosts and calibrate the distance ladder. In Sec. V, we analytically and numerically 
investigate the properties of unscreened main-sequence stars. As expected, they are more luminous than their screened counterparts due to the increased rate of nuclear burning needed to maintain hydrostatic equilibrium when their self-gravity is stronger. In Sec. VI, we study the tip of the red giant branch (TRGB), which is used as a distance indicator. We find that the TRGB distance to unscreened galaxies is overestimated, and thus comparing TRGB and Cepheid distances (which underestimate the distance) is a promising method of constraining our screening mechanism. In Sec. VII, we discuss white dwarfs and type Ia supernovae in unscreened galaxies. The Chandrasekhar mass is smaller when the value of $G$ is increased, while the rescaled supernovae peak luminosity is larger. In Sec. VIII, we study low-mass dwarf stars which could potentially be unscreened in the outskirts of the MW where the dark matter density is lower than it is locally. In particular, we derive the effects of increasing $G$ on the minimum mass for hydrogen burning (separating red and brown dwarfs) and the radius plateau in the brown dwarf Hertzsprung-Russell diagram. The minimum hydrogenburning mass decreases, and the brown dwarf radius is smaller, as one would expect for more compact objects. In Sec. IX, we discuss possible tests of C.2 in the form of mass vs light comparisons. Both strong and weak lensing of extragalactic sources can be used to probe $\gamma$ by comparing with suitable dynamical tracers such as the $\mathrm{x}$-ray surface brightness or stellar velocity dispersion. Finally, we summarize our findings, discuss avenues for future work, and conclude in Sec. X. At times, we will make reference to polytropic models and the Eddington standard model; a brief review of these is provided in Appendix.

Although our tests are framed in terms of the dark matter-baryon interaction model, they are all designed simply to look for signatures of varying Newton's constant outside the solar system. Thus, they apply equally to any theory of gravity in which $G$ varies spatially.

\section{A. Conventions}

We use the mostly plus convention for the Minkowski metric, $\eta_{\mu \nu}=\operatorname{diag}(-1,1,1,1)$. We use $G_{\mathrm{N}}$ to refer to the value of Newton's constant measured locally (in the Solar System) and $G\left(\rho_{\text {DM }}\right)$ to refer to its value at general $\rho_{\text {DM }}$. The Planck mass, which appears in the Einstein-Hilbert action, is $M_{\mathrm{pl}}^{2}=(8 \pi G)^{-1}$; i.e., $G$ without any stated density dependence is a constant. In all cases, $\log (x) \equiv$ $\log _{10}(x)$ unless otherwise stated.

\section{DARK MATTER-BARYON INTERACTIONS}

Here, we briefly review the model proposed by Ref. [30]. In this model, dark matter-baryon interactions give rise to an effective space-time for the baryons that depends on the dark matter density. The dark matter is modeled as an irrotational fluid. The effective field theory for this is simply a $P(X)$ theory $^{2}$ for a scalar $\Theta$ with $X=$ $-g^{\mu \nu} \partial_{\mu} \Theta \partial_{\nu} \Theta$. [Note that we take $\Theta$ to have dimensions of length and $P(X)$ to have dimensions of [mass $]^{4}$.] The action for this sector (including gravity) is

$$
S=\int \mathrm{d}^{4} x \sqrt{-g}\left[\frac{R(g)}{16 \pi G}+P(X)\right]
$$

which gives the energy-momentum tensor for dark matter,

$$
T_{\mu \nu}^{\mathrm{DM}}=2 P_{X} \partial_{\mu} \Theta \partial_{\nu} \Theta+P g_{\mu \nu},
$$

where $P_{X} \equiv \mathrm{d} P(X) / \mathrm{d} X$. From this, we can identify the four-velocity, pressure, and density of the dark matter:

$$
\begin{aligned}
u_{\mu} & =-\frac{\partial_{\mu} \Theta}{\sqrt{X}}, \quad P_{\mathrm{DM}}=P(X), \\
\text { and } \rho_{\mathrm{DM}} & =2 P_{X} X-P(X) .
\end{aligned}
$$

Since we are interested in pressureless nonrelativistic dark matter, we can take $P_{\mathrm{DM}} \ll \rho_{\mathrm{DM}}$, which implies that

$$
\rho_{\mathrm{DM}} \approx 2 P_{X} X \text {. }
$$

The dark matter-baryon interactions arise because the baryons move on an effective Jordan frame metric $\tilde{g}_{\mu \nu}$ that is a combination of the Einstein frame metric $g_{\mu \nu}$ and the dark matter variables. In particular,

$$
\begin{aligned}
\tilde{g}_{\mu \nu} & =R^{2}(X)\left(g_{\mu \nu}+u_{\mu} u_{\nu}\right)-Q^{2}(X) u_{\mu} u_{\nu} \\
& =R^{2}(X) g_{\mu \nu}+S(X) \partial_{\mu} \Theta \partial_{\nu} \Theta,
\end{aligned}
$$

where $R(X)$ and $Q(X)$ are free functions and

$$
S(X) \equiv \frac{R^{2}(X)-Q^{2}(X)}{X} .
$$

The dark matter-baryon interaction is incorporated in the model by augmenting the action (4) with a term

$$
S_{\mathrm{b}}\left[\tilde{g},\left\{\psi_{\mathrm{b}, i}\right\}\right]=\int \mathrm{d}^{4} x \sqrt{-\tilde{g}} \mathcal{L}_{b}\left(\tilde{g},\left\{\psi_{\mathrm{b}, i}\right\}\right),
$$

where $\psi_{\mathrm{b}, i}$ represent the various baryon fields. Cosmic acceleration is achieved as follows. Considering a flat Friedmann-Lemaître-Robertson-Walker universe given by

$$
\mathrm{d} s^{2}=-\mathrm{d} t^{2}+a^{2}(t) \mathrm{d} \vec{x}^{2}
$$

in the Einstein frame, the field $\Theta$ is time dependent so that, using Eq. (7), any factor of $X$ or $\dot{\Theta}$ can always be replaced

\footnotetext{
${ }^{2}$ In general, one needs three scalars $\Phi^{I}$ to describe a fluid, but in the absence of vorticity, there is only one d.o.f., and so the theory is dual to a $P(X)$ theory [51].
} 
by a function of $\rho_{\mathrm{DM}}(t)$. This metric is decelerating since at late times $a(t) \sim t^{2 / 3}$ (recall there is no dark energy in this model). The Jordan frame metric is then

$$
\mathrm{d} \tilde{s}^{2}=-Q^{2}\left(\rho_{\text {DM }}\right) \mathrm{d} t^{2}+R^{2}\left(\rho_{\text {DM }}\right) a^{2}(t) \mathrm{d} \vec{x}^{2} .
$$

The functions $R$ and $Q$ are chosen such that, at late times, i.e., $\rho_{\mathrm{DM}} \sim H_{0}^{2} / G_{\mathrm{N}}$, the scale factor in this frame, $\tilde{a}(\tilde{t})=R\left(\rho_{\mathrm{DM}}(t(\tilde{t})) a(t(\tilde{t}))\right.$, is accelerating. [The time coordinate has been changed from $t$ to $\tilde{t}$ by defining $\mathrm{d} \tilde{t}=Q\left(\rho_{\mathrm{DM}}\right) \mathrm{d} t$.] Thus, cosmic acceleration is achieved without the need for any dark energy d.o.f. At early times, the standard cosmological history is recovered by assuming that both $R$ and $Q$ tend to unity so that $\tilde{g}_{\mu \nu} \rightarrow g_{\mu \nu}$. Since these functions describe the interaction of dark matter with baryons, this must be a density-dependent effect that suppressed deviations from GR when $\rho_{\mathrm{DM}} \gtrsim H_{0}^{2} / G_{\mathrm{N}}$. This observation led the authors of Ref. [30] to speculate that a similar mechanism would screen the interactions on smaller scales, inside collapsed objects. In the next section, we show that this is indeed the case.

\section{SCREENING MECHANISM}

In this section, we derive C.1 and C.2, namely that Newton's constant and the PPN parameter $\gamma$ are density dependent in this theory. (The reader interested only in astrophysical tests can safely skip to the subsequent sections.) We accomplish this by calculating the weakfield limit for an isolated star immersed in a background dark matter density. We assume that the dark matter density does not vary spatially over length scales shorter than the radius of the star. This means that we can treat $X$ as constant and perturb $\Theta=\bar{\Theta}-\theta$ so that $X=\dot{\bar{\Theta}}^{2}-2 \dot{\bar{\Theta}} \dot{\theta}+\mathcal{O}\left(\theta^{2}\right)$. The four-velocity of the dark matter is then (ignoring postNewtonian corrections)

$$
u^{\mu}=\left(1+\mathcal{O}\left(\dot{\theta}^{2}\right), \frac{\partial^{i} \theta}{\dot{\bar{\Theta}}}\right),
$$

from which we can identify the dark matter velocity $v_{\mathrm{DM}}^{i}=\partial^{i} \theta / \dot{\bar{\Theta}}$. Since the dark matter is nonrelativistic, this is $\ll 1$. We assume further that the star itself does not source an appreciable amount of dark matter (i.e., dark matter does not cluster inside the star), so we simply need to solve the Einstein equations for a baryon source. ${ }^{3}$ These are

\footnotetext{
${ }^{3}$ In fact, this assumption can be relaxed without altering the results of our derivation. One can see this by noting that for pure disformal transformations the scalar $\theta$ sourced at Newtonian order only gives post-Newtonian corrections and hence cannot contribute to any fifth force [25]. Additionally, the conformal coupling contributes terms of order $\dot{\bar{\Theta}}$ and $\theta^{\prime 2}$ to the Jordan frame metric, which are both higher order in the post-Newtonian expansion and therefore does not contribute to any fifth force.
}

$$
G_{\mu \nu}=8 \pi G T_{\mathrm{b}, \mu \nu},
$$

where $T_{\mathrm{b}}^{\mu \nu}=2 / \sqrt{-g} \delta S_{\mathrm{b}} / \delta g_{\mu \nu}$. Now, since the baryons couple to dark matter, this is not covariantly conserved $\left(\nabla_{\mu} T_{\mathrm{b}}^{\mu \nu} \neq 0\right)$. It is the Jordan frame energy-momentum tensor $\tilde{T}_{\mathrm{b}}^{\mu \nu}=2 / \sqrt{-\tilde{g}} \delta S_{\mathrm{b}} / \delta \tilde{g}_{\mu \nu}\left(\sqrt{-\tilde{g}}=Q R^{3} \sqrt{-g}\right)$ that is covariantly conserved (with respect to the Jordan frame metric, $\tilde{\nabla}_{\mu} \tilde{T}_{\mathrm{b}}^{\mu \nu}=0$ ). This means that quantities such as the baryon pressure and density should be defined in the Jordan frame using $\tilde{T}_{\mathrm{b}}^{\mu \nu}$. They can then be used in Eq. (14) by converting to the Einstein frame energy-momentum tensor as follows [30]:

$$
\begin{aligned}
T_{\mathrm{b} \mu \nu}= & Q R^{3} \tilde{T}_{\mathrm{b}}^{\kappa \lambda} \\
& \times\left[R^{2} g_{\mu \kappa} g_{\mu \lambda}+\left(2 R R_{X} g_{\kappa \lambda}+S_{X} \partial_{\kappa} \Theta \partial_{\lambda} \Theta\right) \partial_{\mu} \Theta \partial_{\nu} \Theta\right] .
\end{aligned}
$$

In order to calculate this for our nonrelativistic setup, we need to specify a coordinate system. By virtue of our choice of four-velocity in Eq. (13), we are working in Minkowski space in Cartesian coordinates, i.e.,

$$
\mathrm{d} s^{2}=-\mathrm{d} t^{2}+\mathrm{d} x^{2},
$$

at the background level. As we are only interested in calculating the weak-field limit to Newtonian order $\left[\mathcal{O}\left(v^{2} / c^{2}\right)\right]$, it is sufficient to calculate the Jordan frame metric to zeroth order to compute the energy-momentum tensor. At this order, one has

$$
\mathrm{d} \tilde{s}^{2}=-Q^{2} \mathrm{~d} t^{2}+R^{2} \mathrm{~d} x^{2} .
$$

This implies that

$$
\tilde{T}_{\mathrm{b}}^{\mu \nu}=\operatorname{diag}\left(\frac{\tilde{\rho}_{\mathrm{b}}}{Q^{2}}, 0,0,0\right)
$$

where $\tilde{\rho}_{\mathrm{b}}$ represents the Jordan frame density and we have assumed that the baryons are pressureless (i.e., the nonrelativistic limit).

Now, we are in a position to calculate the source for Eq. (14) using Eq. (15):

$$
\begin{gathered}
T_{\mathrm{b} 00}=\frac{R^{3}}{Q}\left[R^{2}+\dot{\bar{\Theta}}^{2}\left(S_{X} \dot{\bar{\Theta}}^{2}-2 R R_{X}\right)\right] \tilde{\rho}_{\mathrm{b}} \\
T_{\mathrm{b} 0 i}=-\frac{R^{3}}{Q}\left[S_{X} \dot{\bar{\Theta}}^{2}-2 R R_{X}\right] \tilde{\rho}_{\mathrm{b}} \dot{\bar{\Theta}} \partial_{i} \theta \\
T_{\mathrm{b} i j}=\frac{R^{3}}{Q}\left[S_{X} \dot{\bar{\Theta}}^{2}-2 R R_{X}\right] \tilde{\rho}_{\mathrm{b}} \partial_{i} \theta \partial_{j} \theta .
\end{gathered}
$$

Since $\partial_{i} \theta \sim v_{i}$, the $0 i$ - and $i j$-components are at a higher post-Newtonian order than the 00-component, and we can 
safely neglect them. ${ }^{4}$ The important point is that we can invert Eq. (7) to write $\bar{X}=\dot{\bar{\Theta}}^{2}$ and $\dot{\bar{\Theta}}$ as a function of $\rho_{\text {DM }}$, which means that the source for the Einstein equations is a function of the local dark matter density. In particular, we can expand Einstein's equations assuming the Newtonian limit for the metric

$$
g_{\mu \nu}=(-1+2 \Phi) \mathrm{d} t^{2}+(1+2 \Psi) \delta_{i j} \mathrm{~d} x^{i} \mathrm{~d} x^{j}
$$

to find

$$
\nabla^{2} \Phi=\nabla^{2} \Psi=-4 \pi G f\left(\rho_{\mathrm{DM}}\right) \tilde{\rho}_{\mathrm{b}}
$$

with

$$
f\left(\rho_{\mathrm{DM}}\right)=\frac{R^{3}}{Q}\left[R^{2}+\dot{\bar{\Theta}}^{2}\left(S_{X} \dot{\bar{\Theta}}^{2}-2 R R_{X}\right)\right] .
$$

The solution of Eq. (23) is

$$
\Phi=\Psi=f\left(\rho_{\mathrm{DM}}\right) \frac{G}{r} \int \mathrm{d}^{3} \vec{x} \tilde{\rho}_{\mathrm{b}}
$$

Typically, one would identify the integral with the star's mass $M$ (or, in the case of the interior, the mass enclosed within a sphere of radius $r$ ), but in our case, there is a subtlety. Because $\tilde{\rho}_{\mathrm{b}}$ is defined in the Jordan frame, we actually have to use the volume element defined using the Jordan frame metric. For this reason, one has

$$
M=\int \mathrm{d}^{3} \vec{x} R^{3} \tilde{\rho}_{\mathrm{b}}
$$

and therefore Eq. (25) is

$$
\Phi=\Psi=\frac{f\left(\rho_{\mathrm{DM}}\right)}{R^{3}} \frac{G M}{r} .
$$

Using this, we can calculate the Jordan frame metric ${ }^{5}$

\footnotetext{
${ }^{4}$ Note that there are two velocities here $-v_{i}$, the dark matter velocity, and the velocity of the baryon fluid-so really this is a post-Newtonian expansion in both velocities. The latter velocity is post-Newtonian by definition, so we are implicitly assuming that the velocity of the dark matter is of the same order or less than the baryon fluid velocity.

${ }^{5}$ Note that there is a contribution to the $0 i$-component of the form $\tilde{g}_{0 i}=S \dot{\bar{\Theta}}^{2} v_{\mathrm{DM} i}$. This can be moved to the 00 -component by performing a linearized gauge transformation $x^{\prime \mu}=x^{\mu}+\xi^{\mu}$ with $\xi_{i}=0$ and $\xi_{0}=-S \dot{\bar{\Theta}}^{2} v_{\mathrm{DM} i} x^{i}[24,25]$. This adds a term $-2 S \dot{\bar{\Theta}}^{2} v_{\mathrm{DM} i} v_{\mathrm{b}}^{i}$ to the 00-component, which we have neglected. It would be interesting to investigate the effects of this (non-PPN) term for the dynamics of test bodies in this space-time. We leave this for future work.
}

$$
\begin{aligned}
\mathrm{d} \tilde{s}^{2}= & \tilde{g}_{\mu \nu} \mathrm{d} x^{\mu} \mathrm{d} x^{\nu} \\
= & Q^{2}\left(-1+2 \frac{f\left(\rho_{\text {DM }}\right)}{R Q^{2}} \frac{G M}{r}\right) \mathrm{d} t^{2} \\
& +R^{2}\left(1+2 \frac{f\left(\rho_{\text {DM }}\right)}{R^{3}} \frac{G M}{r}\right) \delta_{i j} \mathrm{~d} x^{i} \mathrm{~d} x^{j} .
\end{aligned}
$$

The simplest way to extract the observable quantities is to change gauge to quasi-Cartesian coordinates. This can be accomplished by defining

$$
\tilde{t}=Q t \quad \text { and } \quad \tilde{x}^{i}=R x^{i} .
$$

One then finds

$$
\begin{aligned}
\mathrm{d} \tilde{s}^{2}= & \left(-1+2 \frac{G_{\mathrm{N}}\left(\rho_{\mathrm{DM}}\right) M}{\tilde{r}}\right) \mathrm{d} \tilde{t}^{2} \\
& +\left(1+2 \gamma\left(\rho_{\mathrm{DM}}\right) \frac{G_{\mathrm{N}}\left(\rho_{\mathrm{DM}}\right) M}{\tilde{r}}\right) \delta_{i j} \mathrm{~d} \tilde{x}^{i} \mathrm{~d} \tilde{x}^{j}
\end{aligned}
$$

where the gravitational constant

$$
G\left(\rho_{\mathrm{DM}}\right)=\frac{f\left(\rho_{\mathrm{DM}}\right)}{Q^{2}} G
$$

We remind the reader that this is solely a function of $\rho_{\mathrm{DM}}$ because we can always express $\dot{\bar{\Theta}}$ in terms of this. We can also identify the PPN parameter

$$
\gamma\left(\rho_{\mathrm{DM}}\right)=\frac{Q^{2}}{R^{2}}
$$

which controls light bending and the Shapiro time-delay effect. It is also a function of $\rho_{\mathrm{DM}}$. GR predicts that this is unity, and in the Solar System, deviations are constrained to be smaller than $2 \times 10^{-5}$ by the Cassini measurement of the Shapiro time delay [52]. Since we do not have similar measurements in lower-density unscreened galaxies, this bound does not apply in general.

A few comments are in order. First, note that the theory violates the equivalence principle between baryons and dark matter; i.e., baryons and dark matter will fall at different rates in externally sourced gravitational fields if the external source is baryonic in nature. This breaking of the equivalence principle indicates the presence of a fifth force between baryons mediated by a particle with spin different from 2 (i.e., not a graviton) since the equivalence principle is a necessary feature of spin-2 theories [53]. Second, note that when the Jordan frame metric is maximally conformal, i.e., $S=0(Q=R)$, one has $G_{\mathrm{N}}=$ $R^{2} G$ and $\gamma=1$ as one would expect. ${ }^{6}$ Third, in the

\footnotetext{
${ }^{6}$ One would have $\gamma \neq 1$ if $R$ were a function of $\Theta$ rather than $X$ because the baryon fluid would source the scalar. Since we have assumed this is not the case, we expect $\gamma=1$. See Ref. [6] for more details.
} 
TABLE I. Summary of astrophysical probes of the screening mechanism (Secs. IV-IX).

\begin{tabular}{lll}
\hline \hline Probe & \multicolumn{1}{c}{ Effect } & \multicolumn{1}{c}{ Tests } \\
\hline $\begin{array}{l}\text { Cepheid stars } \\
\text { Main-sequence stars }\end{array}$ & $\begin{array}{l}\text { Modification of period-luminosity relation } \\
\text { Luminosity proportional to } G\left(\rho_{\mathrm{DM}}\right)^{3} \text { (low mass) } \\
\text { or } G\left(\rho_{\mathrm{DM}}\right) \text { (high mass) }\end{array}$ & $\begin{array}{c}\text { Compare distance indicators } \\
\text { Hertzsprung-Russell diagram, } \\
\text { globular clusters } \\
\text { Compare distance indicators }\end{array}$ \\
$\begin{array}{l}\text { Tip of the red giant branch } \\
\text { Red dwarf stars }\end{array}$ & $\begin{array}{c}\text { Minimum hydrogen-burning mass proportional } \\
\text { to } G\left(\rho_{\mathrm{DM}}\right)^{1.398}\end{array}$ & $\begin{array}{c}\text { Hydrogen-burning lines in atmosphere } \\
\text { Brown dwarf stars }\end{array}$ \\
$\begin{array}{l}\text { Decrease in radius proportinal to } G\left(\rho_{\mathrm{DM}}\right)^{-1 / 2} \\
\text { at fixed mass }\end{array}$ & Measurement of radius plateau \\
\hline \hline
\end{tabular}

maximally disformal case $R=1$, the results are identical to those derived for pure disformal couplings by Ref. [25]. Finally, one could derive the other PPN parameters by extending the calculation here. We expect that this will be significantly more complicated due to the necessity of including both the dark matter velocity and post-Newtonian baryon fluid variables. It is also likely that one will need to perform a complicated gauge transformation once the Jordan frame metric has been found (see Ref. [25]).

Equations (31) and (32) are the main results of this section. Reference [30] did not give canonical forms for $R$ and $Q$ such that they can drive the cosmic acceleration. Since we wish to remain agnostic, we, too, will not provide functional forms but, rather, will simply treat Newton's constant and $\gamma$ as functions of $\rho_{\mathrm{DM}}$. This allows for the possibility of model-independent tests of the theory that can be used to constrain dark energy (or other) models if and when they are devised. Our only demands will be that $G\left(\rho_{\text {loc }}\right)=G_{\mathrm{N}}$ and that $\gamma\left(\rho_{\text {loc }}\right)$ satisfies the Cassini bound, where $\rho_{\text {loc }}$ is the dark matter density at the location of the Solar System in the Milky Way's halo, approximately $10^{7} M_{\odot} \mathrm{kpc}^{-3}[54,55]$. This ensures that the Solar System is screened so that any tests of this theory are necessarily extragalactic and must be performed using galaxies with dark matter halos that are less dense than the MW. Depending on the form of $G\left(\rho_{\mathrm{DM}}\right)$, it is also possible that the outskirts of the MW are unscreened.

Having identified the novel screening mechanism, the rest of this paper is devoted to finding astrophysical probes of it. We speculate on other potential tests in Sec. X B. Since we require the MW to be screened, we focus on objects that are abundantly observed in other galaxies. We will focus primarily on Cepheid variable stars, both because they can be modeled numerically and because their use as distance indicators has previously been successful in constraining screening mechanisms that only exhibit effects in other galaxies. We will also discuss white dwarf stars and type Ia supernovae, as well as main-sequence stars, the tip of the red giant branch, and low-mass stars (red and brown dwarfs).

In this work, we will take the threshold for screening to be the ambient dark matter in the Solar System, $\rho_{\text {loc }}=10^{7} M_{\odot} \mathrm{kpc}^{-3} \cdot{ }^{7}$ Objects in regions denser than this have $G\left(\rho_{\mathrm{DM}}\right)=G_{\mathrm{N}}$, whereas those in regions less dense than this have $G\left(\rho_{\text {DM }}\right)>G_{\mathrm{N}},{ }^{8}$ with $\Delta G$ a constant independent of $\rho_{\text {DM }}$. Since $G\left(\rho_{\text {DM }}\right)$ does not vary over the objects we consider, we can then derive the change in these objects by setting

$$
G\left(\rho_{\mathrm{DM}}\right)=\left(1+\frac{\Delta G}{G_{\mathrm{N}}}\right) G_{\mathrm{N}}
$$

It is important to bear in mind that in general the value of $\Delta G$ is likely to vary between objects inhabiting regions of different dark matter densities.

The reader uninterested in the technical details of the tests should consult Table I and may then skip to Sec. X.

\section{CEPHEID VARIABLE STARS AND THE PERIOD-LUMINOSITY RELATION}

Cepheid variable stars have proven to be useful tools for constraining screening mechanisms $[4,7,37,42]$. In this section, we introduce Cepheids and the PLR and calculate the change to both when Newton's constant is varied. Our discussion will be agnostic as to the cause of $G \neq G_{\mathrm{N}}$; in practice, the precise model for $G$ would be input at a later stage when comparing the predictions made here with observations.

\section{A. Cepheid stars}

Cepheids are post-main-sequence stars of mass approximately $3-20 M_{\odot}$ that pulsate with a period that is dependent primarily upon their luminosity with a weak dependence on mass and metallicity. They are composed of

\footnotetext{
${ }^{7}$ This is the cosmological dark matter density at $z \approx 40$, which may imply that this model predicts that deviations from $\Lambda \mathrm{CDM}$ emerge around this epoch or later since it is possible that the threshold dark matter density is lower than in the MW.

${ }^{8}$ Note that we have implicitly assumed that the fundamental theory functions are such that the strength of gravity is enhanced in low-density environments. One could instead have $G<G_{\mathrm{N}}$, in which case the results of the tests studied in this work would be opposite. For example, main-sequences stars would be less luminous.
} 


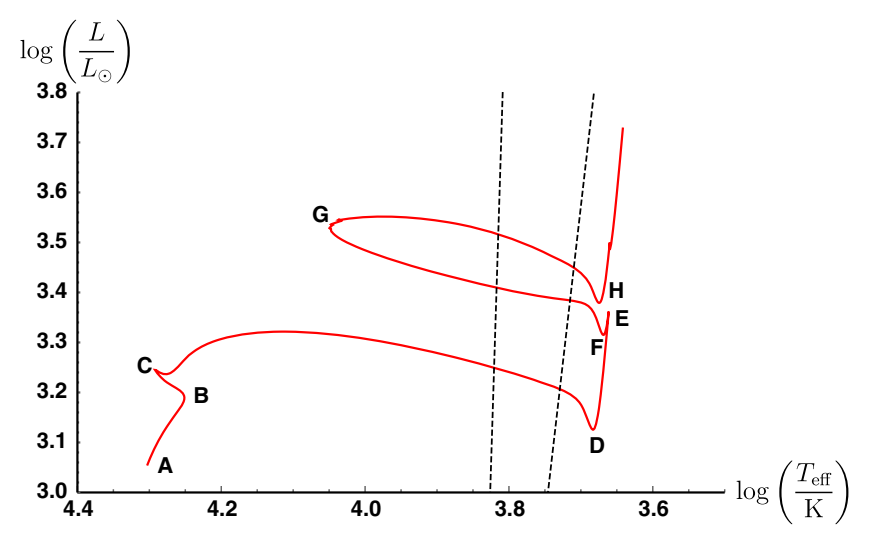

FIG. 1. The evolution of a $5 M_{\odot}$ star with $Z=0.0006$ in GR (solid, red). The dashed black lines show the edges of the instability strip. (A) ZAMS. (B) Core contraction begins. (C) Central hydrogen exhaustion. (D) Envelope becomes convective. (E) Central hydrogen burning begins. (F) Blue loop begins. (G) Farthest point on the blue loop. (H) Central helium exhausted.

a mainly inert convective helium core (there is a small amount of helium burning at the very center of the core) surrounded by a thin hydrogen-burning shell that is their primary power source. Surrounding this is a convective envelope. The envelope contains a thin layer of partially ionized helium that drives the pulsations. In particular, a small contraction of the star will cause a rise in the nuclear burning rate, which is accompanied by a temperature increase. This would typically result in an increase in the outward pressure due to photon absorption in the outer layers, but in these stars, it instead causes further helium ionization, which causes an increase in the opacity. This makes further ionization easier, leading to further contractions. This ionization zone therefore acts as an energy dam, storing energy that is eventually released, resulting in the outward phase of the pulsation. This opacity-driven process is known as the $\kappa$-mechanism [56].

Figure 1 shows the typical evolution of a Cepheid star from its zero age main sequence (ZAMS) (point A) through central helium exhaustion (point $\mathrm{H}$ ); we have chosen a $5 M_{\odot}$ star, but any mass will exhibit qualitatively similar features. The $\kappa$-mechanism can only drive pulsations in the narrow region between the two dashed lines referred to as the instability strip. This is because the internal stellar motion must be nonadiabatic in order for the ionization zone to store energy. If it is not, then the energy losses above the ionization zone would necessarily balance the energy gains at its base. The blue edge of the instability strip corresponds to the stage of stellar evolution where the ionization zone enters the nonadiabatic region, and the red edge corresponds to the stages where convective motion damps the pulsations sufficiently that they are essentially quenched. Examining the figure, one can see that a star will cross the instability strip at least three times [it is possible to have more crossings between (E) and (H)]. The first crossing occurs between phases (C) and (D) and lasts for a very short time (approximately $10^{4} \mathrm{yr}$ ), making the probability of observing stars here extremely low. The second crossing and third crossing occur during phases $(\mathrm{F})-(\mathrm{H})$ during the so-called blue loop, which lasts approximately 1-100 Myr, and it is on these crossings that Cepheids are typically observed.

The blue loops are the result of the interplay of several complex physical processes that are not fully understood [57-59]. Indeed, Fig. 1 shows the track for a star that has executed a blue loop, but the size, shape, and even existence of the loop are highly dependent on the prescription one employs for these processes. For this reason, it will prove instructive to understand the salient features governing each phase of evolution in Fig. 1 to aid us in constructing models that exhibit blue loops when $G\left(\rho_{\mathrm{DM}}\right) \neq G_{\mathrm{N}}$. We caution that our review will be far from comprehensive, and we refer the reader to the exhaustive literature (e.g., Ref. [60]) on this ongoing field of research for more in-depth discussions.

Point (A) is the ZAMS. At this point, the star begins to burn hydrogen in its core, depleting its supply and producing helium. At point (B), the hydrogen core begins to contract. This is the first place where internal processes affect the post-main-sequence dynamics. The amount of contraction and overshooting will determine the final mass and radius of the core, as well as the hydrogen and helium gradients above the core. Point (C) corresponds to core hydrogen depletion. At this point, the core is composed of inert helium surrounded by a thick hydrogen-burning shell. A large fraction of the energy generated by this shell is absorbed by the outer layers of the star, causing a rapid expansion and cooling of the envelope. This is the reason for the reddening of the star in the color-magnitude diagram (CMD). This phase spanning the region between (C) and (D) lasts approximately $10^{4} \mathrm{yr}$. This is the second phase where internal processes are important. The efficiency of convective mixing and the length scale over which convective mixing occurs plays an important role in determining the temperature and luminosity of point (D). Smaller mixing lengths move it to lower temperatures. Point (D) is the Hayashi track. This is the point where the envelope has become fully convective. The core temperature during this phase can exceed the ignition temperature for the triplealpha process, so some helium burning occurs in the core, but the star's luminosity is primarily due to hydrogen burning in the thin shell surrounding the core. The star is a red giant between (D) and (E). Point (E) is referred to as the dredge-up. The convective envelope has extended into the region that was formerly the convective core during mainsequence burning so that material that has been processed through the Carbon-Nitrogen-Oxygen cycle is mixed throughout the entire envelope.

The blue loop may begin at point $(\mathrm{F})$, when the envelope becomes radiative. The blue-loop phase is well studied 
numerically and is a generic feature of many numerical codes (e.g., Refs. [61-63]), but it is not the result of any one single simple physical process. Whether or not the star executes a blue loop depends on the size of the core, the amount of overshooting (overshooting increases the helium core mass at the end of hydrogen burning, and outward overshooting from the core can inhibit the loops, whereas inward shooting from the base can exacerbate them [57]), and the amount of convective mixing. Mass loss and rotation can also be important. One essential requirement for the execution of the loops is efficient semiconvection [59]. There are two inequivalent criteria for convection: the Schwarzschild criterion and the Ledoux criterion (see, e.g., Ref. [64]). In homogeneous media, the two are equivalent, but in the presence of strong gradients in the chemical composition, it is possible that the Schwarzschild criterion is violated but the Ledoux criterion is not. In such semiconvective regions, mixing is slow. For Cepheid stars at stage $(F)$, the amount of semiconvection determines the efficiency with which helium is mixed into the core. Point (G) is the hottest part of the blue loop, and point $(\mathrm{H})$ indicates the end of core helium burning where the star rejoins the Hayashi track. All of the processes described above lack a completely fundamental description and are typically implemented into stellar structure codes using phenomenological descriptions controlled by efficiency parameters that are likely mass dependent. These are used to understand the role and importance of these processes for various stages of stellar evolution.

For stars with 3-9 $M_{\odot}$, stellar modeling indicates that the blue loops are abundant, provided that semiconvection is included in the model. For heavier stars $\left(10-20 M_{\odot}\right)$, the situation is more complicated [59]. For these stars, core helium burning can begin before point $D$ in Fig. 1. Whether or not this happens depends strongly on the mixing processes described above, and it is possible that the star can end up as a red supergiant or a blue supergiant or spend some time in both phases (blue loops). For these stars, the existence of blue loops is highly dependent on the choice of the efficiency parameters for the mixing processes.

\section{B. Period-luminosity relation}

When a star crosses the instability strip to become a Cepheid, it obeys a well-known relationship between pulsation period, $P$, and luminosity, $L$ [65],

$$
\log (L)=A \log (P)+\epsilon \log \left(T_{\text {eff }}\right)+\beta
$$

with $A \simeq 1.3$ [66]. Heuristically, this relationship comes about as follows. Using stellar perturbation theory, one can show that the period of any stellar oscillation satisfies $[42,56,67]$

$$
P \propto \sqrt{\frac{R^{3}}{G_{\mathrm{N}} M}} .
$$

Using the Stefan-Boltzmann law, $L \propto R^{2} T_{\text {eff }}^{4}$, we can eliminate the radius in favor of the luminosity and the effective temperature, and we can further eliminate the mass $M$ by assuming a mass-luminosity relation. ${ }^{9}$ Thus, we are left with a relation between the period, the effective temperature, and the luminosity, which can always be written in the form of Eq. (34). In practice, the coefficients $A, \epsilon$, and $\beta$ must be fit to data rather than derived from first principles.

\section{Unscreened period-luminosity relation}

We now wish to understand how the PLR changes in galaxies less dense than the MW that may be unscreened so that $G\left(\rho_{\mathrm{DM}}\right)>G_{\mathrm{N}}$. Examining Eqs. (34) and (35), one can discern two potential effects. First, since $P \propto G\left(\rho_{\mathrm{DM}}\right)^{-1 / 2}$, increasing $G\left(\rho_{\mathrm{DM}}\right)>G_{\mathrm{N}}$ reduces the period; i.e., the star pulsates faster. The second effect is that we expect the luminosity at fixed mass to be larger. It is well documented that increasing the strength of gravity makes stars more luminous at fixed mass due to an increase in the nuclear burning rate that is necessary to produce the pressure gradient needed to sustain hydrostatic equilibrium (see Ref. [7] and references therein). The change to the PLR can be written as

$\Delta \log (L)=\frac{A}{2} \log \left(1+\frac{\Delta G}{G_{\mathrm{N}}}\right)+B(M) \log \left(1+\frac{\Delta G}{G_{\mathrm{N}}}\right)$,

where $\Delta \log (L) \equiv \log (L)-\log \left(L_{\mathrm{GR}}\right)$ is the difference between the true luminosity and that which would be inferred if one had used the GR relation given in Eq. (34). The term proportional to $A$ is the effect of changing the pulsation period [see Eq. (35)], whereas the second is an ansatz parametrizing the change in the luminosity due to the increased rate of nuclear burning. We will justify this post hoc by showing that a power-law relation is a good approximation. It is possible that the free coefficient $B$ is a function of the stellar mass, so we have included this possibility. We will also find the value of $B$ to depend on whether the Cepheid is observed at the second or third crossing of the instability strip.

In order to calculate $B$, we have modified the stellar structure code MESA $[61-63,68]$ to compute stellar evolution for general $G\left(\rho_{\text {DM }}\right)$. We have evolved a grid of $5 M_{\odot}-13 M_{\odot}$ models with $Z=0.0006$ from the premain-sequence through the blue loop phase in order to

\footnotetext{
${ }^{9}$ There is some residual mass and metallicity dependence since a core mass-radius relation is more appropriate for shell-burning stars.
} 

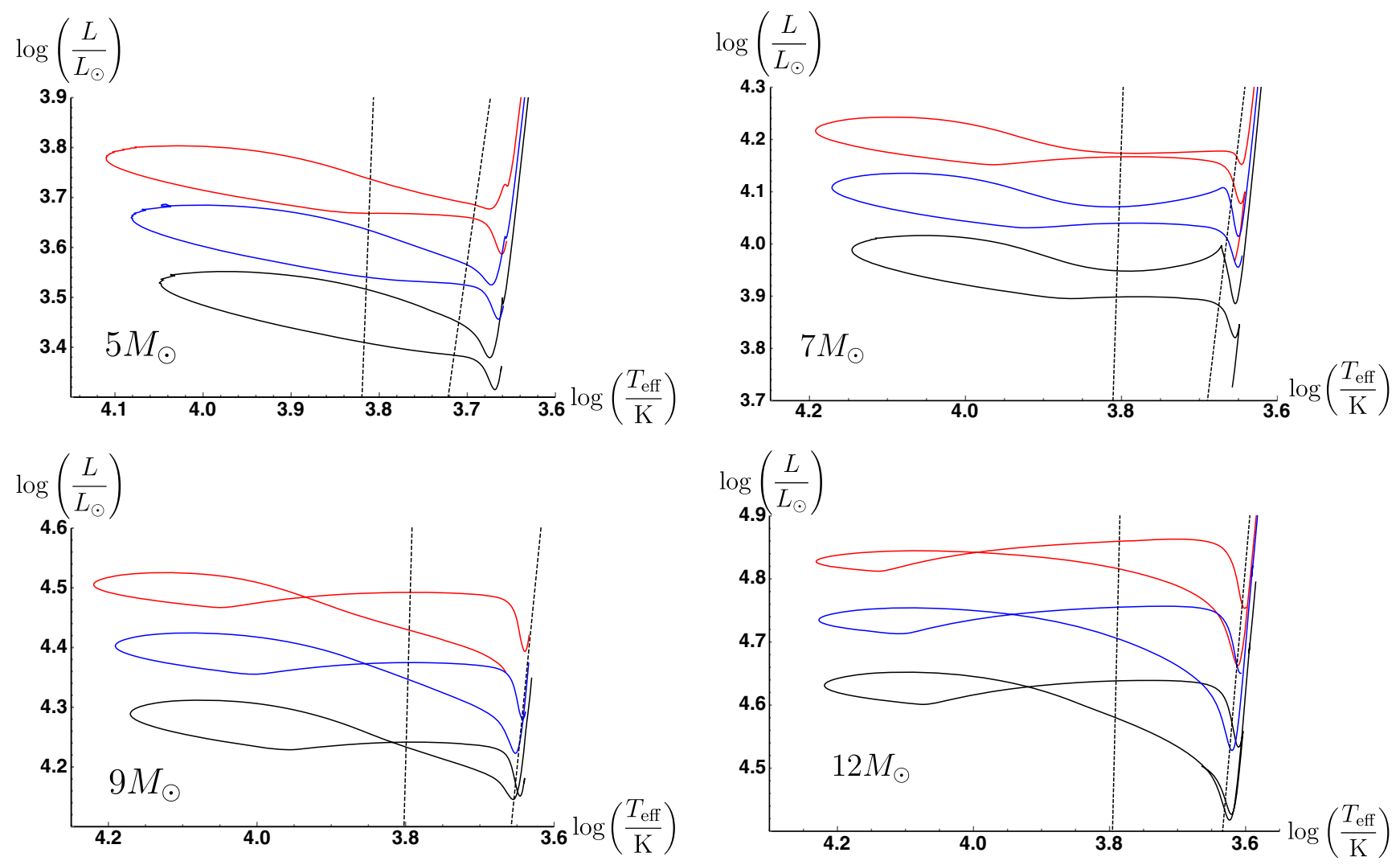

FIG. 2. The blue loops for representitive stellar models used to derive the coefficients $B(M)$ given in Table II, which appear in the PLR in Eq. (36). The black (lower) lines show GR models $\left(\Delta G / G_{\mathrm{N}}=0\right)$, and the blue (middle) and red (upper) lines correspond to $\Delta G / G_{\mathrm{N}}=0.075$ and $\Delta G / G_{\mathrm{N}}=0.15$, respectively. The black dashed lines show the edges of the instability strip. Each panel corresponds to a different mass, as indicated. Tracks for other masses are qualitatively similar.

calculate $\Delta \log (L)$. We varied $\Delta G / G_{\mathrm{N}}$ in the range $0 \leq \Delta G / G_{\mathrm{N}} \leq 0.15$. For masses $3 M_{\odot}<M<9 M_{\odot}$, we found the loops to be generic, provided that semiconvection and the Ledoux criterion are enabled. This allowed us to use a fixed set of mixing parameters. We chose the mixing length parameter to be $\alpha_{\mathrm{MLT}}=1.73\left(\alpha_{\mathrm{MLT}} \equiv \lambda_{\mathrm{MLT}} / H_{P}\right.$, where $\lambda_{\mathrm{MLT}}$ is the mixing length and $H_{P}$ is the pressure scale height). The efficiency of semiconvection was taken to be $\alpha_{S C}=0.1$. For overshooting, we adopted the parameters $f=0.014$ and $f_{0}=0.004$ for burning and nonburning cores of all compositions. See the instrumentation papers [61-63,68] and [69] for the details of the overshooting parameters. For masses in the range $10 M_{\odot}-13 M_{\odot}$, we found the loops to be uncommon (as expected Ref. [59]), and it was necessary to vary the mixing parameters as a function of $\Delta G$ in order to produce models that exhibited them. We used $1.1 \leq \alpha_{\mathrm{MLT}}<1.3$ and $10^{-4}<$ $\alpha_{\mathrm{SC}}<100$ in order to achieve this. Overshooting was as above. Different parameter choices lead to small variations in the blue-loop shape and luminosity at fixed mass, which is part of the scatter in the PLR that is present even in GR. Ideally, this uncertainty would be included in any statistical analysis that applies the PLR we derive here to find observational bounds on $\Delta G$.
The resultant loops for some representative masses are shown in Fig. 2 (for visual clarity, we do not show the preloop phase). The loops for other masses not shown are qualitatively similar. We have calculated the coefficients $B(M)$ given in Eq. (36) at each crossing of the blue edge of the instability strip (this is a convenient comparison point, and the physics is better understood here) by performing a linear fit for each mass. The coefficients are given in Table II, and visual representations of our models and the

TABLE II. Slope $B(M)$ of the $\Delta \log (L)-\log \left(1+\Delta G / G_{\mathrm{N}}\right)$ relation given in Eq. (36) for a range of Cepheid masses measured at second or third crossing of the instability strip.

\begin{tabular}{lcc}
\hline \hline$M / M_{\odot}$ & $B$ at second crossing & $B$ at third crossing \\
\hline 5 & 4.21 & 3.67 \\
6 & 4.52 & 3.61 \\
7 & 4.45 & 3.79 \\
8 & 4.34 & 3.58 \\
9 & 4.18 & 3.46 \\
10 & 4.00 & 3.48 \\
11 & 3.81 & 3.58 \\
12 & 3.67 & 3.92 \\
13 & 3.58 & 3.95 \\
\hline \hline
\end{tabular}



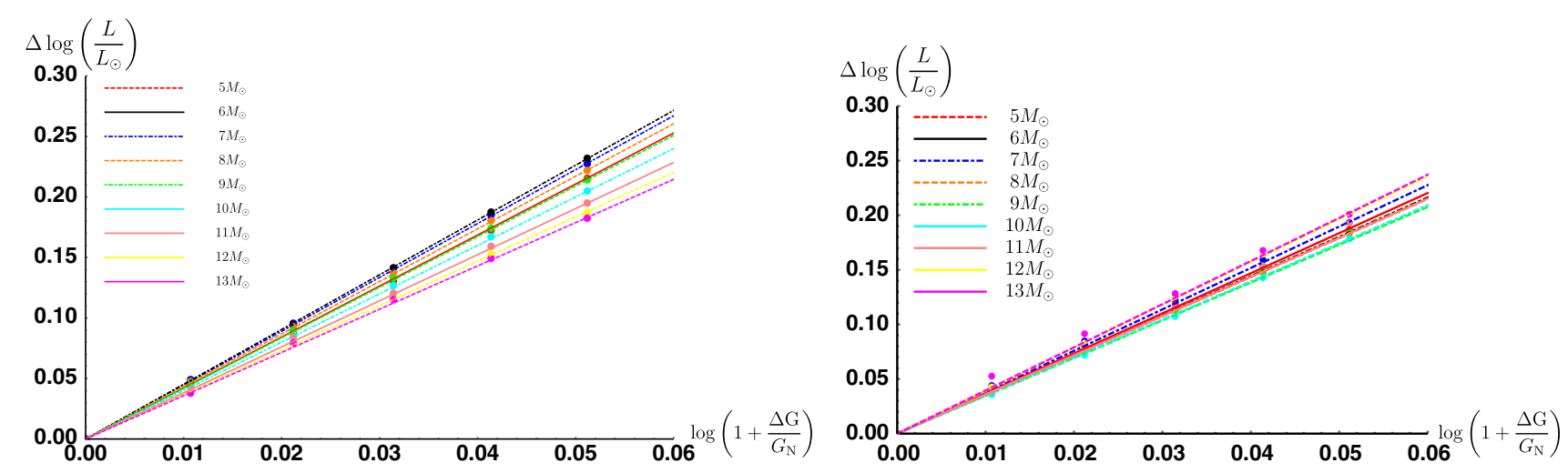

FIG. 3. $\Delta \log (L)$ as a function of $\log \left(1+\Delta G / G_{\mathrm{N}}\right)$ at the blue edge of the instability strip for the second crossing (left) and the third crossing (right). The points indicate the results of MESA models, and the lines are the best-fit linear relations, the slopes of which are the coefficients $B(M)$ given in Table II.

best-fitting relations are given in Fig. 3. Future observational tests of this screening mechanism using unscreened galaxies (with dark matter densities smaller than the MW) can utilize these coefficients to make the necessary theoretical predictions to constrain the theory.

\section{MAIN-SEQUENCE STARS}

Main-sequence stars have been less successful at constraining screening mechanisms than post-main-sequence stars, primarily because they are subject to degeneracies that are more difficult to break. For example, fifth force effects such as luminosity enhancements are degenerate with the metallicity [39]. ${ }^{10}$ One promising test is that they may produce novel deviations in the galactic properties as a whole as a result of their integrated effects [36], so we briefly study their properties here. We will do this both analytically and numerically (using MESA). Both approaches have previously been used in the literature to derive the properties of main-sequence stars in other screening models [36,39], and Ref. [71] has studied the effects of changing the fundamental constants on some stellar properties.

\section{A. Analytic expectations}

The Eddington standard model is a simple analytic model for main-sequence stars. It is certainly not detailed enough to produce accurate models for individual objects, but it has proven useful in the study of screened stars for discerning the main effects of changing fundamental physics on the gross stellar properties [36,39]. This is precisely because the lack of detailed microphysics and time evolution allows one to isolate the effects of changing

\footnotetext{
${ }^{10}$ The one exception to all this is the Sun, where we have precision measurements of quantities such as the solar neutrino flux and access to thousands of oscillation modes. Constraints from the Sun at the level of $\Delta G / G_{\mathrm{N}} \sim 10^{-2}$ have been obtained by considering the effects on the seismic solar model [70], but these do not apply to theories where the Solar System is screened.
}

$G$. Its use in understanding how screening mechanisms affect main-sequence stars is standard in the literature $[3,36]$, so we will not reproduce its derivation in detail, but we do provide a brief review for the unfamiliar reader in Appendix. There, we introduce polytropic stellar models and derive the formulas we use in this section.

The main result of the Eddington standard model is an analytic expression for the luminosity as a function of mass [Eq. (A19)]

$$
L=\frac{4 \pi(1-\beta(M)) G_{\mathrm{N}} M}{\kappa},
$$

where $\kappa$ is the (assumed constant) opacity and $\beta(M)$ is the ratio of the gas pressure to the total pressure [the ratio of the radiation pressure to the total pressure is $1-\beta(M)$ ]. $\beta(M)$ is found by solving a quartic equation given in Eq. (A16). Stars that are entirely gas supported have $\beta=1$ so that there is no luminosity, ${ }^{11}$ and stars which have $\beta=0$ are entirely radiation supported, and the luminosity is maximum (the Eddington luminosity). A careful analysis of Eq. (A16) reveals that $\beta(M)$ is a decreasing function of mass so that low-mass stars are primarily gas supported, whereas high-mass stars are primarily radiation supported. One can use simple scaling arguments to show that the luminosity of gas-supported stars scales as $G^{4} M^{3}$ and the luminosity of radiation-supported stars scales like $G M$ $[3,36]$. In the context of screening mechanisms, this implies that low-mass stars are more sensitive probes of fifth forces than high-mass stars.

To investigate this quantitatively, we simply need to change $G_{\mathrm{N}} \rightarrow G_{\mathrm{N}}(1+\Delta G / G)$ in Eq. (37). ${ }^{12}$ There are two places where this replacement must be made: in Eq. (37)

\footnotetext{
${ }^{11}$ This is a drawback of the approximations made in Eddington standard model. Setting $\beta=1$ is tantamount to removing photons from the star entirely.

${ }^{12} \mathrm{An}$ analysis similar to the one performed here first appeared in the unpublished lecture notes at http://www.jeremysakstein .com/astro_grav_2.pdf by one of the authors.
} 


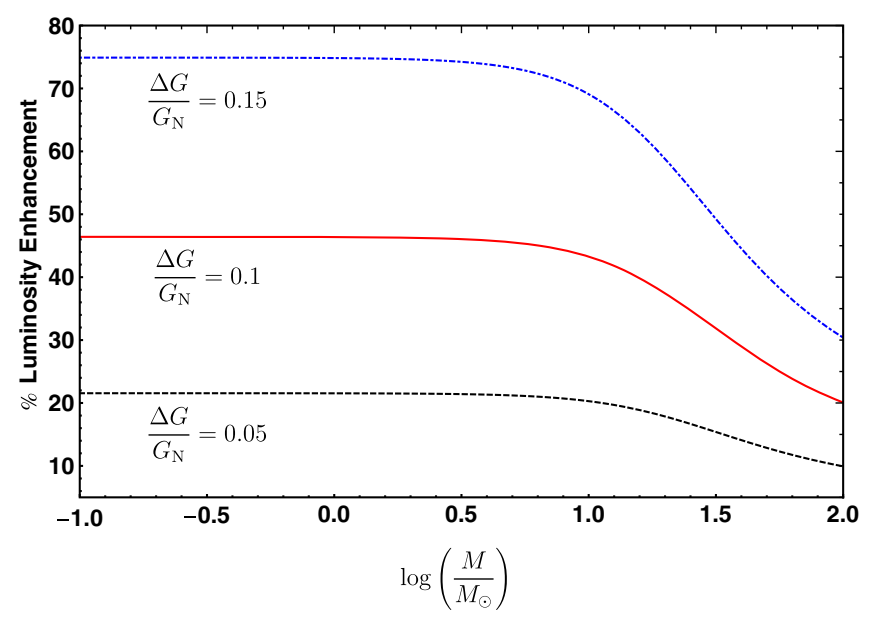

FIG. 4. Analytical predictions (computed using the Eddington standard model) for the luminosity enhancement of unscreened main-sequence stars compared with screened stars of identical masses. The values of $\Delta G / G_{\mathrm{N}}$ are indicated below the corresponding curves.

directly and in Eddington's quartic equation (A16) [see Eq. (A17)]. The second replacement results in the following quartic equation,

$$
\frac{1-\beta(M, \Delta G)}{\beta^{4}(M, \Delta G)}=\left(1+\frac{\Delta G}{G_{\mathrm{N}}}\right)^{3}\left(\frac{M}{M_{\mathrm{Edd}}}\right)^{2}
$$

where the Eddington mass $M_{\text {edd }} \approx 18.2 \mu^{-2} M_{\odot}(\mu$ is the mean molecular weight) is defined in Eq. (A17). The ratio of the luminosity of unscreened and screened stars is then

$$
\frac{L_{\text {unscreened }}}{L_{\text {screened }}}=\left(1+\frac{\Delta G}{G_{\mathrm{N}}}\right) \frac{1-\beta(M, \Delta G)}{1-\beta(M, 0)} .
$$

We are interested in fifth force models where $\Delta G / G_{\mathrm{N}}>0$. We therefore expect that unscreened stars are more luminous than screened stars at fixed mass since these stars require a faster nuclear burning rate in order to maintain hydrostatic equilibrium given the greater inward gravitational force. More photons are therefore produced as a byproduct. We have solved Eq. (38) numerically for $\Delta G / G_{\mathrm{N}}$ in the range $0-0.15$ and have used the resulting function to calculate the luminosity enhancement using Eq. (39). Our results are plotted in Fig. 4, where it is evident that unscreening main-sequences stars indeed enhances the luminosity. Furthermore, in line with our discussion above, the enhancement is indeed larger in low-mass starstending to the factor of $\left(1+\Delta G / G_{\mathrm{N}}\right)^{4}$ that we predicted above - since these are gas pressure supported and therefore more sensitive to $G$. The factor of approximately $75 \%$ for $\Delta G / G_{\mathrm{N}} \sim 0.15$ is likely a gross overestimate, given that the many assumptions underlying this model greatly simplify the internal dynamics of the star and neglect many important physical processes. This is why it is of paramount importance to use numerical simulations to account for these deficiencies, which we do in the next subsection.

\section{B. Numerical models}

In order to verify our expectations above, we have evolved a grid of solar-mass and two-solar-mass models with solar metallicity $(Z=0.02)$ for $\Delta G / G_{\mathrm{N}}$ in the range $0 \leq \Delta G / G_{\mathrm{N}} \leq 0.15$. The evolutionary tracks in the CMD are shown in Fig. 5. Evidently, the main sequence is indeed increasingly luminous (at fixed evolutionary point) when $\Delta G / G_{\mathrm{N}}$ is increased, as is the red giant phase. In some cases, increasing $\Delta G / G_{\mathrm{N}}$ can make the tracks appear to mimic those that would be exhibited by larger-mass objects when $\Delta G / G_{\mathrm{N}}=0$ (i.e., GR). For example, comparing the track for $\Delta G / G_{\mathrm{N}}=0.15$ in the left panel with the track for $\Delta G / G_{\mathrm{N}}=0$ in the right, its shape is more akin to that of a $2 M_{\odot}$ star than a solar mass object. (The star in the left panel is cooler and less luminous than a $2 M_{\odot}$ star, so the
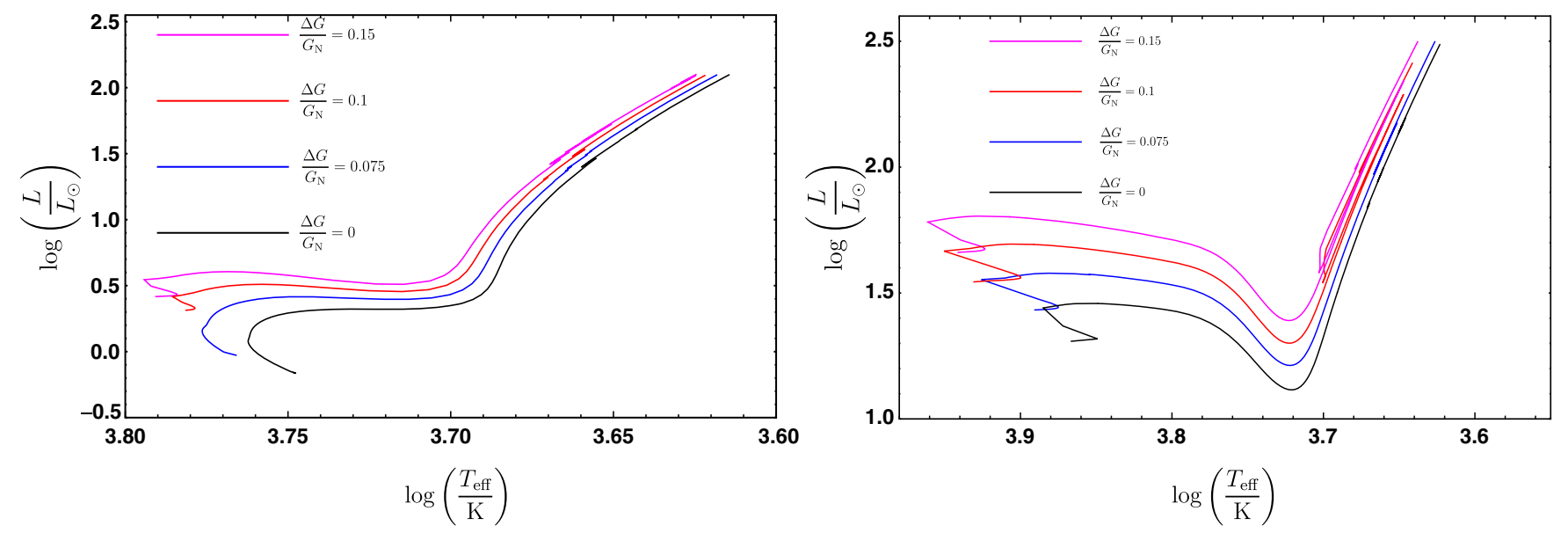

FIG. 5. Main-sequence evolution for a $1 M_{\odot}$ (left) and $2 M_{\odot}$ (right) star of solar metallicity $\left(Z=0.02\right.$ ) for values of $\Delta G / G_{\mathrm{N}}$ as indicated in the figure. 

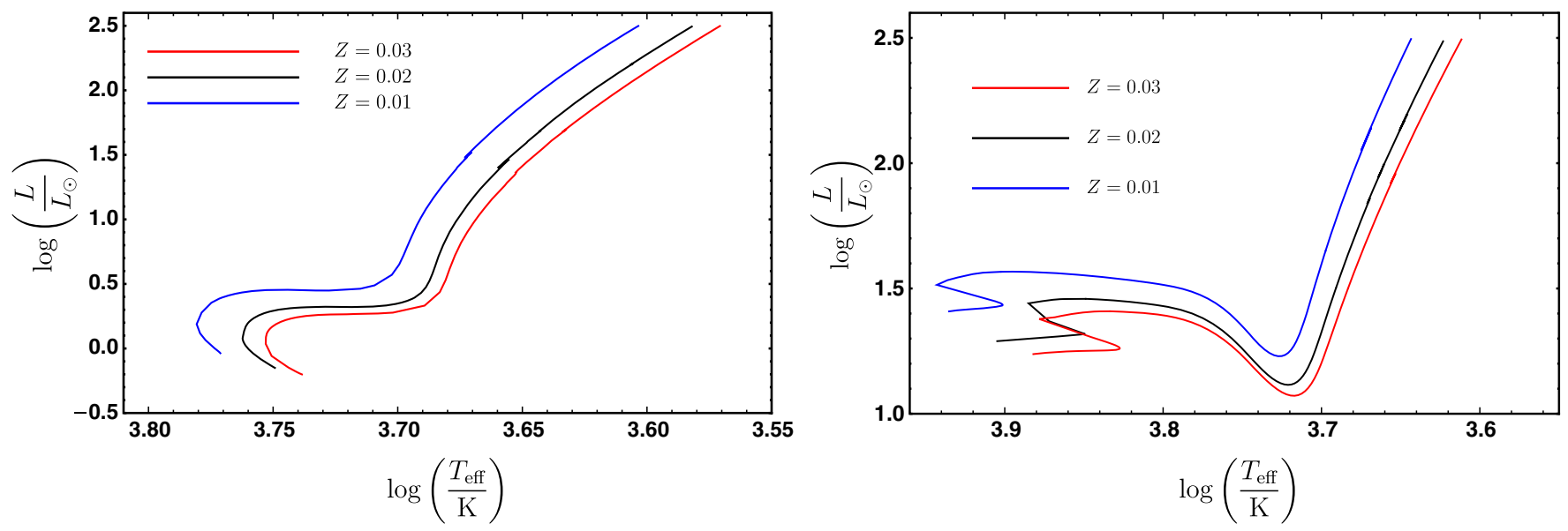

FIG. 6. The evolution of solar mass (left) and $2 M_{\odot}$ (right) stars when $\Delta G / G_{\mathrm{N}}=0$ but the metallicity $Z$ is varied as indicated in the figure.

exact object it is mimicking in GR would have a mass smaller than $2 M_{\odot}$.) This makes modified gravity partially degenerate with the initial mass function in setting the overall photometric properties of galaxies.

Unlike the Cepheid PLR, which has only a small scatter from metallicity effects, the main-sequence luminosity is very sensitive to the metallicity. This is one reason that main-sequence stars have been less successful at constraining theories that exhibit screening mechanism than post-main-sequence and dwarf stars. Any observational test based on the results derived here must mitigate this degeneracy either by measuring the metallicity or by marginalizing over it with a suitable prior. To illustrate this degeneracy, we plot the evolutionary tracks in the CMD for one- and two-solar-mass stars in GR with $Z=$ 0.01 (subsolar) and $Z=0.03$ (supersolar) metallicity in Fig. 6. It is clear that metallicity is an important degeneracy for the main sequence.

\section{POST-MAIN-SEQUENCE STARS AND THE TIP OF THE RED GIANT BRANCH}

One powerful method for constraining theories where the strength of gravity varies between galaxies is to compare different distance estimates to the same galaxy [37]. Since different indicators are sensitive to $G$ to varying degrees, the distances will only agree if one has the correct theory of gravity. We have already derived the change in the Cepheid PLR, so in this section, we will study another distance indicator that has previously been used to constrain modified gravity theories: the tip of the red giant branch.

When stars of mass $0.9 \lesssim M / M_{\odot} \lesssim 2$ exhaust their central hydrogen, they leave the main sequence and evolve along the red giant branch. At this point, they are composed of a degenerate isothermal ${ }^{4} \mathrm{He}$ core surrounded by a thin shell of hydrogen in hydrostatic equilibrium. Hydrogen fusion in this shell is solely responsible for the star's luminosity. As the helium core contracts, both the core's and the shell's temperatures increase. When the temperature exceeds that necessary to ignite helium burning, the triple- $\alpha$ process begins, and the star moves onto the asymptotic giant branch in a very short time. This leaves a visible discontinuity in the I-band magnitude at $I=4 \pm 0.1$ with a very small scatter due to metallicity effects [65]. By looking for this discontinuity, the distance to the star can be calculated since both the magnitude and flux are known.

The effects of changing $G$ on the TRGB were investigated by Ref. [37]. Increasing $G$ has the effect of increasing the rate of hydrogen shell burning around the core since a faster rate is needed to balance the stronger gravity. This has the effect of causing the shell and core temperature to rise at a faster rate, igniting the triple- $\alpha$ process earlier, which causes the luminosity at the tip to decrease. Using MESA, we have investigated this effect for our screening mechanism by evolving $1.3 M_{\odot}$ stars of solar metallicity $(Z=0.02)$ to the TRGB. Our results are plotted in Fig. 7. The left panel shows the Hertzsprung-Russell diagram for some representative values of $\Delta G / G_{\mathrm{N}}$. One can see that low values produce similar tip luminosities, whereas stronger modifications produce drastic reductions. This is quantified in the right panel where we plot the ratio of the tip luminosity to the GR value as a function of $\Delta G / G_{\mathrm{N}}$. This is well fit by the relation

$\frac{L_{\mathrm{TRGB}}}{L_{\mathrm{TRGB}, \mathrm{GR}}}=1.0019\left[1-0.00189148\left(1+\frac{\Delta G}{G_{\mathrm{N}}}\right)^{13.4866}\right]$

Using the equation relating flux and luminosity distance, $F=L / d_{L}^{2}$, one can see that the lower tip luminosity for unscreened stars will result in the GR formula overestimating the distance: 

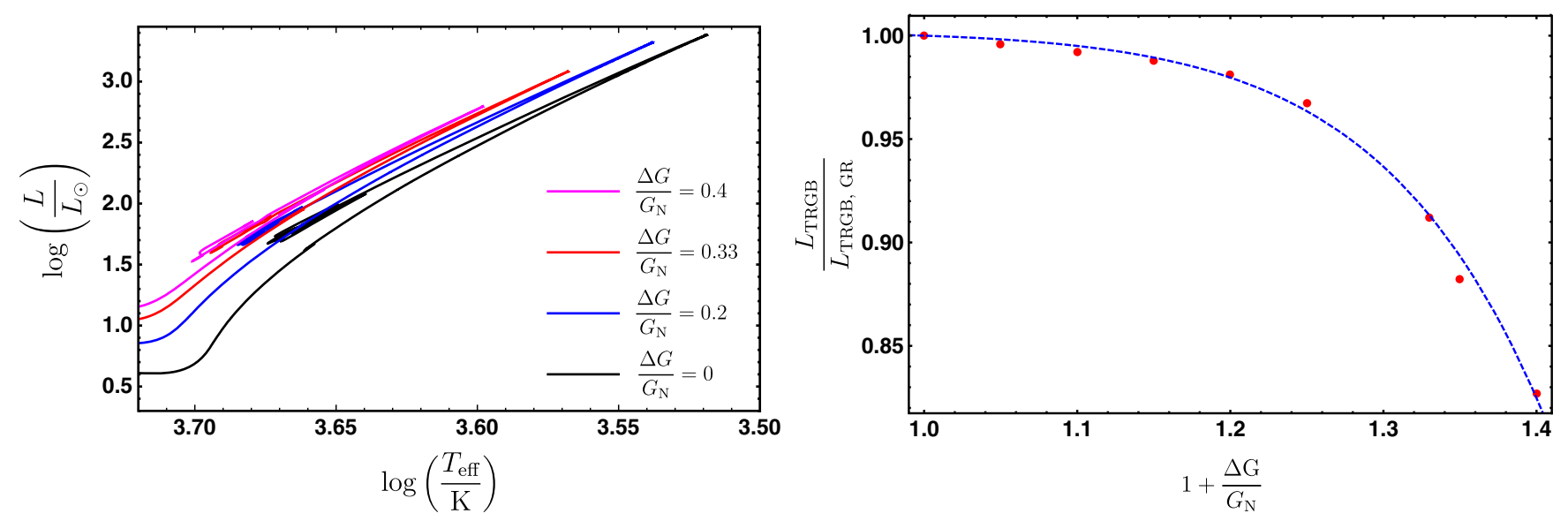

FIG. 7. Left: The Hertzsprung-Russell diagram for $1.3 M_{\odot}, Z=0.02$ stars for values of $\Delta G / G_{\mathrm{N}}$ indicated in the figure. Each curve terminates at the TRGB. Right: The luminosity of the TRGB compared with the GR value, as a function of $1+\Delta G / G_{\mathrm{N}}$. The red points are numerical MESA models, and the blue dashed line is the fitting function of Eq. (40).

$$
d_{L}=d_{L}^{\mathrm{GR}} \sqrt{\frac{L_{\mathrm{TRGB}}}{L_{\mathrm{TRGB}, \mathrm{GR}}}} .
$$

These results indicate that comparing TRGB distances with other indicators, in particular Cepheids, which we have already demonstrated may be unscreened for some parameter choices, is a promising test of this mechanism. We perform this test quantitatively in a companion paper [72].

\section{WHITE DWARFS AND TYPE IA SUPERNOVAE}

Type Ia supernovae are standardizable candles, which has made them a powerful tool for cosmology. The progenitors of type Ia supernovae are white dwarf stars that exceed their Chandrasekhar mass and undergo a thermonuclear runaway. The primary variable determining the peak luminosity of the light curve is the mass of nickel56 available for the explosion. A simple approximation is that the white dwarf is composed entirely of ${ }^{56} \mathrm{Ni}$ so that the mass of nickel is equal to the Chandrasekhar mass $M_{\mathrm{Ch}}$. White dwarfs close to the Chandrasekhar limit are very accurately described by a gas of relativistic particles, which have equation of state $P=K \rho^{\frac{4}{3}}$; i.e., the equation of state is polytropic with index $n=3$ (see Appendix where we provide a brief introduction to polytropic stars).

The Chandrasekhar mass is derived in Eq. (A9) where one can see that $M_{\mathrm{Ch}} \propto G_{\mathrm{N}}^{-\frac{3}{2}}$. The equivalent expression for unscreened white dwarfs can be found by rescaling $G_{\mathrm{N}} \rightarrow G_{\mathrm{N}}\left(1+\Delta G / G_{\mathrm{N}}\right)$. The ratio of the two is

$$
\frac{M_{\mathrm{Ch}}(\Delta G)}{M_{\mathrm{Ch}}}=\left(1+\frac{\Delta G}{G_{\mathrm{N}}}\right)^{-\frac{3}{2}} .
$$

The naive expectation is then that unscreened type Ia supernovae have lower peak luminosities than their screened doppelgängers, owing to their lower Chandrasekhar masses, which implies less ${ }^{56} \mathrm{Ni}$. This conclusion is indeed naive. Reference [73] has studied the effects of changing the strength of gravity on type Ia supernovae. Using a semianalytic model, they found that after one accounts for the variation of the mass of ${ }^{56} \mathrm{Ni}$ with the total mass, and the standardization procedure where the light curve is stretched to match a standard template, the ultimate effect of strengthening gravity is to enhance the peak luminosity. In the context of our model, this implies that the distance to unscreened supernovae is underestimated if one does not account for this enhancement. Fitting Fig. 7 (left) of Ref. [73], one finds relation

$$
\frac{L_{\text {peak }}(\Delta G)}{L_{\text {peak }, \mathrm{GR}}}=\left(1+\frac{\Delta G}{G_{\mathrm{N}}}\right)^{C}
$$

with $C \simeq 1.46$. This formula can be used in future observational probes of (for example) screened dark matterbaryon interactions involving type Ia supernovae, either using observations of individual events or by comparing different distance ladder measurements.

\section{LOW-MASS STARS}

Dwarf stars have proven to be powerful probes of gravity theories where the gravitational constant varies inside of extended objects $[40,41,74]$. Brown dwarf stars are inert objects that are supported by Coulomb scattering pressure. They are not heavy enough for their cores to reach the temperatures and densities necessary for hydrogen fusion. Red dwarf stars are heavier objects that can achieve the central conditions necessary to ignite hydrogen burning, albeit on a chain that results in the net production of ${ }^{3} \mathrm{He}$ rather than the proton-proton chains (which result in ${ }^{4} \mathrm{He}$ ). The transition mass separating these two objects is known as the minimum mass for hydrogen burning, $M_{\mathrm{Min}}$, and the 

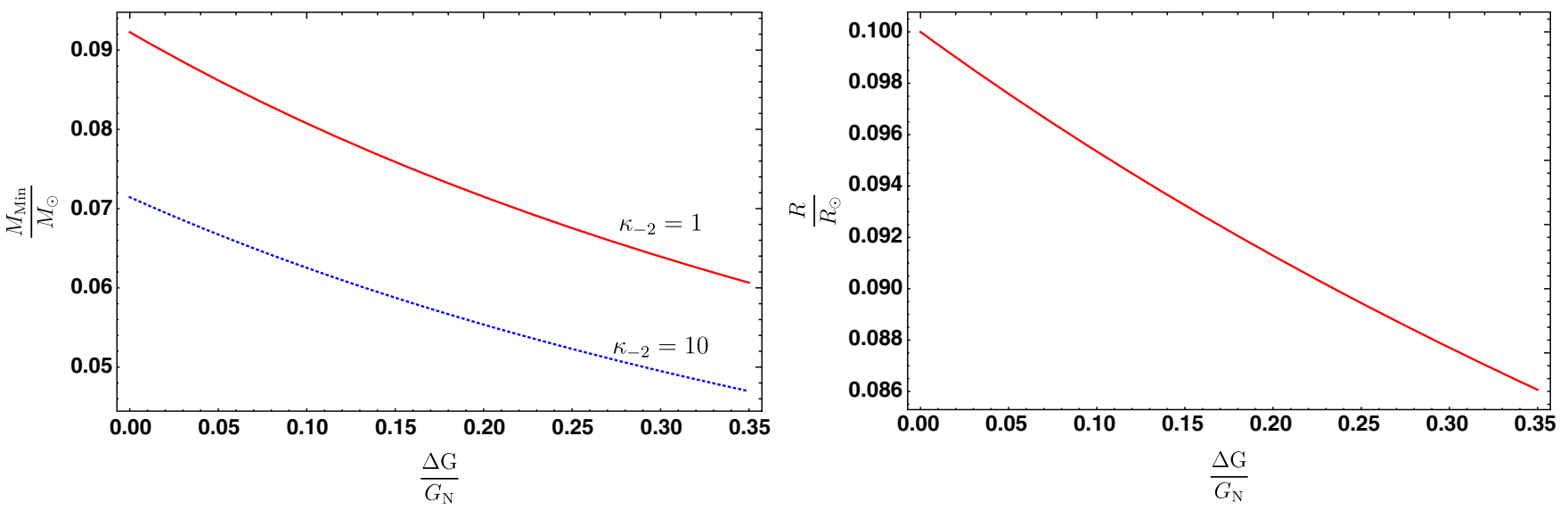

FIG. 8. Left: The minimum mass for hydrogen burning as a function of $\Delta G / G_{\mathrm{N}}$ for two representative opacities given in the figure. Right: The (mass-independent) radius of brown dwarf stars as a function of $\Delta G / G_{\mathrm{N}}$.

relative simplicity of dwarf stars allows for its analytic calculation with few degeneracies. This has made it a powerful probe of cosmologically relevant modified gravity theories $[40,41]$.

The screening mechanism we have derived in this work allows for the possibility that stars in the outskirts our own Galaxy are unscreened since the dark matter density falls below that in the solar neighborhood in these regions. For this reason, in this section, we will study how changing the gravitational constant affects the properties of dwarf stars.

\section{A. Red dwarfs and the minimum mass for hydrogen burning}

The derivation of the minimum mass for hydrogen burning is standard in the literature, so we will not repeat it here. Instead, we will simply focus on the underlying physics, referring the reader to Refs. [40,41,74] for the analytic details. Stable hydrogen burning is achieved when the luminosity due to nuclear fusion in the stellar core balances the luminosity losses from the photosphere. If the gravitational constant increases to values larger than $G_{\mathrm{N}}$, the star will become more compact, raising the central temperature and density. One therefore expects the minimum mass for hydrogen burning to be smaller in unscreened stars than screened stars, or, said another way, it is possible that brown dwarfs in the solar neighborhood could be red dwarfs in the Galactic outskirts. This could potentially change the shape of the HertzsprungRussell diagram measured in globular clusters in the Galactic outskirts.

In order to quantify this effect, we will use the formula derived by Ref. [74], Appendix B2,

$$
\frac{M_{\text {Min }}}{M_{\odot}}=0.092 \kappa_{-2}^{-0.111}\left(1+\frac{\Delta G}{G_{\mathrm{N}}}\right)^{-1.398},
$$

where $\kappa_{-2}$ is the Rosseland mean opacity measured in units of $10^{-2} \mathrm{~g} \mathrm{~cm}^{-2}$. This is the only source of uncertainty in this equation, and it is not very significant. We plot $M_{\min }$ as a function of $\Delta G / G_{\mathrm{N}}$ in the left panel of Fig. 8. In GR, the minimum mass for a red dwarf is $M_{\mathrm{Min}} \sim 0.08 M_{\odot}$. From the figure, one can see that, depending on the opacity and $\Delta G$, in unscreened regions of the Galaxy, stars with masses as low as $0.05 M_{\odot}$ could be hydrogen-burning red dwarfs.

\section{B. Brown dwarfs and the radius plateau}

Stars with masses smaller than $M_{\min }$ are brown dwarfs supported by Coulomb scattering pressure. Objects supported by Coulomb scattering have a fixed radius that is independent of their mass [41]. This leads to a distinctive radius plateau in the Hertzsprung-Russell diagram for lowmass objects. In GR, the radius is given by $R \sim 0.1 R_{\odot}$, but the formula used to derive this contains a factor of $G_{\mathrm{N}}^{-1 / 2}$ [41] so that the radius of unscreened stars scales as

$$
R=0.1\left(1+\frac{\Delta G}{G_{\mathrm{N}}}\right)^{-\frac{1}{2}} R_{\odot}
$$

Physically, increasing Newton's constant makes the star more compact. We plot the radius of brown dwarf stars as a function of $\Delta G / G_{\mathrm{N}}$ in the right panel of Fig. 8. The predicted decrease in radius plateau shown in this figure can potentially be tested with the brown dwarfs observed by GAIA, provided one can measure the radius. If this is the case, one could plot the brown dwarf radius as a function of distance from the Galactic Center; in our model, one might expect to see a negative correlation.

\section{DYNAMICAL VS LENSING MASS}

In this section, we discuss potential tests of the dark matter-density dependence of the PPN parameter $\gamma\left(\rho_{\mathrm{DM}}\right)$ predicted in Eq. (32), focusing on strong and weak gravitational lensing. Both of these rely on the fact that the dynamical mass of an object $M_{\text {Dyn }}$ (measured using dynamical or kinematic tracers) differs from the lensing 
mass $M_{\text {Lens }}$ (measured using gravitational lensing observations) according to

$$
M_{\text {Lens }}=\frac{1+\gamma\left(\rho_{\text {DM }}\right)}{2} M_{\text {Dyn }} \text {. }
$$

\section{A. Strong gravitational lensing}

For this test, typically applicable to clusters and massive elliptical galaxies, the lens mass is determined from the radius of the Einstein ring or multiple image locations. (Note that the dark matter density at the Einstein radius and in galaxy clusters is higher than locally.) The dynamical mass is determined from the velocity dispersion of stars in elliptical galaxies and galaxy clusters or from x-ray emission or the Sunyaev-Zel'dovich signal of the hot gas assumed to be in hydrostatic equilibrium. The most stringent extragalactic bound, $\gamma=0.97 \pm 0.09$ in the galaxy ESO 325-G004, was reported by Ref. [75]. Similarly, Ref. [76] reported $\gamma=1.01 \pm 0.05$. This bound is unlikely to be useful for constraining this screening mechanism because the dark matter density at the location of the lens is likely larger than the screening threshold for the Solar System (the central velocity dispersion of ESO 325-G004, $371 \mathrm{~km} / \mathrm{s}$ implies a dark matter density a few times that of the MW), but similar tests in less dense dark matter environments may be possible.

\section{B. Weak gravitational lensing}

Tests using weak lensing may be more fruitful since one can stack many galaxies to make measurements in less dense environments. Several variants of this test have already been performed in the context of testing gravity. On cluster scales, one can use the x-ray surface brightness as a probe of the dynamical mass, and 30\% bounds on $\gamma$ have been obtained [45,77-79]. Other possibilities on cosmological scales are to cross-correlate weak lensing measurements with redshift space distortions [80] or peculiar velocity measurements [81] or to constrain the $E_{G}$ statistic [82,83]. Applying these to our model would require deriving the equations for linear cosmological perturbations, which will depend on the fundamental theory functions, as well as more precise characterization of the relevant dark matter densities.

\section{CONCLUSIONS}

In this section, we summarize our results, discuss directions for future work, and conclude.

\section{A. Summary of the paper}

In this paper, we have demonstrated that dark matterbaryon interactions can give rise to a new screening mechanism. While Ref. [30] speculated that such a mechanism exists, we have derived it here in detail. We found that there are two consequences of the screening mechanism: the values of both Newton's constant and the PPN parameter $\gamma$ (which describes the relative motion of light and matter) become functions of the local dark matter density. The screening mechanism is present whenever the fundamental functions appearing in the theory are such that the dark matter-baryon interactions vanish in high dark matter densities but become important at lower density. This is what one would expect from any model where dark energy emerges at late times (low cosmological dark matter densities) from such interactions.

Lacking canonical forms for these functions, we adopted a simple approach to understanding the screening mechanism and exploring its observational consequences. The Milky Way must be screened for the theory to pass local tests of GR, so we expect that the underlying parameters of any viable theory are such that objects are only unscreened $\left[G\left(\rho_{\mathrm{DM}}\right)>G_{\mathrm{N}}\right]$ in densities smaller than that at the position of the Solar System. We also assumed that at densities below this threshold $G\left(\rho_{\mathrm{DM}}\right)$ is larger than in the Solar System by a constant amount, i.e., $G\left(\rho_{\mathrm{DM}}\right)=\left(1+\Delta G / G_{\mathrm{N}}\right) G_{\mathrm{N}}$. This allowed us to understand the observational consequences using a one-parameter description of unscreened objects. A more complex dependence on $\rho_{\text {DM }}$ could lead to $\Delta G / G_{\mathrm{N}}$ for astrophysical objects varying between different halos or even within a given halo. It is unlikely that this parameter will vary over the scale of stellar objects, however, since in conventional dark matter models these do not induce large variations in $\rho_{\mathrm{DM}}$. The results of this work can then be considered general, provided that one bears in mind that $\Delta G / G$ may need to be varied on an object-by-object basis even within a single halo.

We next discussed several observational tests. Under our simple model, this is tantamount to understanding how the properties of astrophysical object are altered when one increases Newton's constant by fixed amount. ${ }^{13}$ Since the Solar System must be screened, the most likely tests would be extragalactic. For this reason, we focused on objects in other galaxies of which the properties can be measuredCepheid variable stars and type Ia supernovae. The Cepheid period-luminosity relation in particular is a powerful tool for constraining screening mechanisms $[37,67]$. By running a suite of numerical simulations using a version of the stellar structure code MESA $[61-63,68]$ that was modified to change the value of Newton's constant, we derived the modification to the PLR as a function of $\Delta G / G_{\mathrm{N}}$; under a fifth force, we find a decrease in pulsation period and overall increase in luminosity. We provide the coefficients describing the (mass-dependent) luminosity effect in

\footnotetext{
${ }^{13} \mathrm{~A}$ similar phenomenon occurs in degenerate higher-order scalar tensor (DHOST) theories [74,84]. In this case, the value of Newton's constant is altered inside astrophysical bodies due to a breaking of the Vainshtein mechanism (there is no dependence on the dark matter density). The phenomena discussed in this work could therefore also be used to constrain DHOST theories.
} 
Table II. We found that the distance to unscreened galaxies would be underestimated if one assumed the GR PLR. We also discussed how type Ia supernovae, which are standardizable candles, would behave if they are unscreened, finding that the light curve peak luminosity (after stretch correction) is enhanced.

Using analytic and numerical techniques, we demonstrated that unscreened main-sequence stars are, as expected, more luminous at fixed mass and metallicity than Newtonian or screened stars, although degeneracies with the metallicity will likely make tests using individual objects difficult. The one exception to this is stars observed in our own Galaxy. In particular, globular clusters in the outskirts of the Milky Way may be unscreened due to the smaller dark matter density. There are fewer degeneracies associated with these objects since the metallicity and age can be determined. The luminosity at the tip of the red giant branch was found to be smaller than the GR value, implying that the application of the GR formula will overestimate the true distance. It is thus possible to test our predictions by comparing the TRGB and Cepheid distances to unscreened galaxies, as we do in a companion paper [72].

For ancillary results, we studied how low-mass red and brown dwarf stars respond to an increased Newton's constant. These objects cannot be observed in other galaxies, but it is possible that Milky Way dwarfs are unscreened if they are sufficiently far from the solar neighborhood that the dark matter density is sufficiently low. We found that the minimum mass for hydrogen burning, which separates red from brown dwarfs, decreases for $G>G_{\mathrm{N}}$, so objects with masses as low as $0.05 M_{\odot}$ could be red dwarfs, compared with GR where the threshold is $0.08 M_{\odot}$. We also found that the radius plateau in the brown dwarf Hertzsprung-Russell diagram would shift to smaller radii. Given that GAIA is able to observe these objects, it is possible for these predictions to be tested in the near future. In more complicated models than we have studied here, it is possible that $G\left(\rho_{\mathrm{DM}}\right)<G_{\mathrm{N}}$ in some galaxies. One can use our results to calculate the observable consequences of this; e.g., main-sequence stars would be less luminous than in GR, and the minimum mass for hydrogen burning would be larger.

Finally, we discussed tests of the predicted dark matterdensity dependence of the PPN parameter $\gamma$. Measuring this in other galaxies requires determining both dynamical and lensing mass. Such mass vs light tests has become a standard probe of modified gravity theories, and both weak and strong lensing systems could provide interesting bounds.

\section{B. Future directions}

We have performed a preliminary study of the dark matter-baryon screening mechanism. Here, we discuss future theoretical and observational directions. Since the threshold for screening corresponds to the dark matter density at redshift $z \leq 40$, we expect that at times earlier than this the Universe evolves in a matter identical to the predictions of GR. For this reason, we only discuss latetime phenomenology.

\section{Construction of a canonical model}

We have (deliberately) not chosen any specific form for the free functions describing the dark matter-baryon interactions in this work. Instead, we have treated $G$ and $\gamma$ as generic functions of $\rho_{\mathrm{DM}}$. Choosing forms for these would allow for further tests using observables that are not described simply by a constant shift in $G$. It would be convenient if a canonical model could account for dark energy, but not essential because the screening mechanism may have interesting and potentially useful consequences on smaller scales.

\section{Gravitational waves}

In this theory, gravitational waves move on geodesics of the Einstein frame metric $g_{\mu \nu}$, but light moves on geodesics of the Jordan frame metric $\tilde{g}_{\mu \nu}$, which implies that they may move at different speeds in general. The simultaneous detection of light and gravitational waves from a neutron star merger event (GW170817 [85]) has constrained any difference to be at the $10^{-15}$ level, which has placed strong bounds on modified gravity theories [19-22]. This deviation could be used to constrain the free functions of the present theory. It is highly likely that the combination of parameters that sets the speed of gravitational waves is different from the combinations setting $G\left(\rho_{\mathrm{DM}}\right)$ and $\gamma\left(\rho_{\text {DM }}\right)$, so this strong bound is not necessarily debilitating. It is also possible to construct theories from first principles where the speeds of light and gravity are identical [86-89]. One should check the manner in which such bounds apply and also that the theory is not being applied outside the range of validity of effective field theory [90].

\section{Distance indicator comparisons}

Comparing screened and unscreened distance estimates to the same galaxy has yielded strong bounds on chameleon (and similar) theories [37]. In this work, we have studied both Cepheids and TRGB distance indicators, finding that both are unscreened with the former underestimating the true distance and the latter overestimating it. A test of this mechanism along the lines of Ref. [37] could therefore yield important constraints on $\Delta G / G_{\mathrm{N}}$. We perform this test in a companion paper [72].

\section{Binary pulsars}

Theories that include disformal couplings may predict different values of Newton's constant for nonrelativistic objects relative to gravitational waves. In particular, the orbital decay of binary pulsars is sensitive to $G_{\mathrm{GW}}$ and $c_{T} / c$, the speed of gravitational waves relative to the speed 
of light discussed above [91]. Constraints are at the $10^{-2}$ level, so a calculation of $G_{\mathrm{GW}}$ once a canonical model is specified would be highly constraining.

\section{Hubble tension}

The discrepancy between the Planck central value of the Hubble constant and the value inferred using the distance ladder is currently $4.4 \sigma$ [92], which is strong evidence for new physics affecting one or both of these probes. Unscreening galaxies that calibrate the supernova magnitude-redshift relation could provide a resolution. This is the focus of a companion paper [72].

\section{Baryon fraction in collapsed objects}

The baryon fraction in galaxies and clusters is time dependent if dark matter and baryons redshift at different rates [93]. In this theory, baryons redshift as $a^{-3}$, but dark matter will redshift at a different rate (in a manner dependent on the choice of fundamental theory functions) since it moves on geodesics of a different (Einstein frame) metric. In other words, dark matter and baryons inhabit effective space-times that are expanding at different rates. The theory may therefore be tested by measuring the baryon fraction as a function of redshift.

\section{Cosmological tests}

Once a canonical model is specified, one could derive the equations of motion for the background cosmology and linear cosmological perturbations and use these to constrain the theory using current datasets, for example, cosmic microwave background, baryon acoustic oscillation, and large scale structure data. The nonstandard redshifting of dark matter at late times (discussed in the previous subsection) implies that the amount of dark matter required in the early Universe may differ from the $\Lambda$ CDM value. Thus, further tests using the relic abundance of dark matter and big bang nucleosynthesis may be possible.

\section{Integrated Sachs-Wolfe effect}

The theory predicts that photons interact with dark matter in a manner that causes them to feel the fifth force. This implies that the integrated Sachs-Wolfe (ISW) effect will differ from the GR prediction. Other theories that make similar predictions have been tightly constrained by ISW observations [94,95].

\section{Tests of the weak equivalence principle between baryons and dark matter}

Unscreened baryons feel a fifth force, whereas dark matter does not, so the weak equivalence principle (WEP) is violated. Extragalactic tests of the WEP could constrain this screening mechanism. Indeed, such tests have been fruitful in the context of chameleon screening [38,48$50,96,97]$, although in that case, it is dark matter that is typically unscreened, so one would need to derive the predictions for our theory separately.

\section{Two-body calculations}

The analysis we have performed in this work considered a single isolated object. It would be interesting to extend this to two-body systems. In some cases, a violation of the WEP can emerge [29,98]. The dark matter-baryon interaction is described by a disformal transformation $[24,99,100]$, which is known to exhibit novel effects in many-body systems [101-103].

\section{Tests in the strong-field regime}

Strong-field tests of gravity are a powerful probe of alternatives to GR. Once a canonical model is specified, it would be straightforward to calculate the equivalent of the Tolman-Oppenheimer-Volkov equations for the theory and hence derive the properties of neutron stars in unscreened galaxies.

\section{Dark matter detection experiments}

The authors of Ref. [30] noted that, similarly to how Newton's constant is dark matter-density dependent, Fermi's constant is likewise. This may have implications for dark matter experiments (both direct and indirect), although it is straightforward to see that modifications are screened in high-density environments so the bounds are weak. We refer the reader to Ref. [30] for the details.

\section{Conclusion}

The new screening mechanism that we have studied in this work derives from a dark energy model that is not yet constrained, or even well characterized. The scarcity of dark energy models that are not ruled out or fine-tuned makes this model an interesting candidate for future study. The observational consequences we have derived here could form the basis for new astrophysical probes of the mechanism, which should be supplemented with cosmological tests, tests in the strong-field regime, and other tests described above. The construction of a canonical model with which to benchmark constraints would be a useful first step.

We have suggested several future directions, both theoretical and observational, that could lead to a deeper understanding and further bounds on this screening mechanism. These may ultimately prove too constraining to allow the theory to account for dark energy, as is the case with other screening mechanisms. Even if this were to happen, the screening mechanism would remain of interest in its own right, and regions of parameter space where $G$ varies between galaxies but the theory does not behave as dark energy may still provide interesting intermediate-scale phenomenology. 
Finally, we note that we have focused on devising novel probes of the theory's prediction that Newton's constant varies as a function of the local dark matter density. There are other theories (either fundamental or phenomenological) where $G$ is spatially varying in a manner that is correlated with physical quantities. The tests we have devised in this work apply equally to these theories, provided one correlates $G$ correctly with the variables that govern its variation.

\section{ACKNOWLEDGMENTS}

We are grateful for discussions with Koushik Sen, Radek Smolec, and the wider MESA community for answering our various MESA-related questions. We would like to thank Justin Khoury for several enlightening discussions. J. S. is supported by funds made available to the Center for Particle Cosmology by the University of Pennsylvania. H. D. is supported by St. John's College, Oxford, and acknowledges financial support from ERC Grant No. 693024 and the Beecroft Trust. B. J. is supported in part by the US Department of Energy Grant No. DE-SC0007901 and by NASA ATP Grant No. NNH17ZDA001N.

\section{APPENDIX: STELLAR STRUCTURE MODELS}

In this section, we briefly review polytropic equations of state and spherically symmetric polytropic stars in GR, which are now standard in the literature relating screening mechanisms to stellar structure. Further details can be found in Refs. $[4,36,39] .{ }^{14}$

\section{Polytropic stars}

A polytropic equation of state is one where the pressure

$$
P=K \rho^{\gamma}=K \rho^{\frac{n+1}{n}},
$$

where $K$ is a constant and $n$ is referred to as the polytropic index. When used in conjunction with the hydrostatic equilibrium equation

$$
\frac{\mathrm{d} P}{\mathrm{~d} r}=-\frac{G_{\mathrm{N}} M(r) \rho(r)}{r^{2}}
$$

and the equation of mass conservation

$$
\frac{\mathrm{d} M(r)}{\mathrm{d} r^{2}}=4 \pi r^{2} \rho(r)
$$

the equations of stellar structure become self-similar so that all dimensionful quantities can be scaled out, and the equations can be written purely in terms of dimensionless variables. This is achieved as follows. First, define the dimensionless quantities

\footnotetext{
${ }^{14}$ See also http://www.jeremysakstein.com/astro_grav_2.pdf.
}

$\xi=\frac{r}{r_{c}}, \quad \rho=\rho_{c} \theta(\xi)^{n}, \quad r_{c}^{2}=\frac{(n+1) P_{c}}{4 \pi G \rho_{c}^{2}}$,

where $P_{c}$ and $\rho_{c}$ are the central pressure and density, respectively. Using Eq. (A1), one has $P=\rho_{c} \theta(\xi)^{n+1}$ so that the function $\theta(\xi)$ completely characterizes the pressure and density profiles of the star in terms of the dimensionless radial coordinate $\xi$. [In fact, $\theta(\xi)$ is the dimensionless temperature because $T=T_{c} \theta(\xi)$.] Combining the relations (A4) with the stellar structure Eqs. (A2) and (A3), one finds the Lane-Emden equation

$$
\frac{1}{\xi^{2}} \frac{\mathrm{d}}{\mathrm{d} \xi}\left(\xi^{2} \frac{\mathrm{d} \theta(\xi)}{\mathrm{d} \xi}\right)=-\theta(\xi)^{n}
$$

This can be solved for a given value of $n$ either numerically or, in the cases $n=0,1,5$, analytically. The boundary conditions are $\theta(0)=1\left(\rho(r=0)=\rho_{c}\right)$ and $\theta^{\prime}(0)=0$ [by virtue of spherical symmetry $\mathrm{d} P /\left.\mathrm{d} r\right|_{r=0}=0$, which can be seen from Eq. (A2) by setting $M(0)=0$ ]. The radius of the star is the point where $P(R)=0$. One can find this by integrating Eq. (A5) to the point $\xi_{R}$ such that $\theta\left(\xi_{R}\right)=0$. The radius is then

$$
R=r_{c} \xi_{R}
$$

Substituting Eq. (A5) into Eq. (A3) and integrating from $\xi=0$ to $\xi=\xi_{R}$, one finds an expression for the stellar mass,

$$
M=4 \pi r_{c}^{3} \omega_{R} \rho_{c}=4 \pi \omega_{R}\left[\frac{(n+1) K}{4 \pi G_{N}}\right]^{\frac{3}{2}} \rho_{c}^{\frac{3-n}{2 n}}
$$

where

$$
\omega_{R} \equiv-\left.\xi_{R}^{2} \frac{\mathrm{d} \theta}{\mathrm{d} \xi}\right|_{\xi_{R}}
$$

and we have used Eq. (A4). The analytic models studied in this work can all be described by $n=3$ polytropic models. In this case, according to Eq. (A7), the mass is independent of the central density. For systems comprised of gasses that are nearly relativistic $(\gamma=4 / 3, n=3)$, this represents the limiting, Chandrasekhar mass:

$$
M_{\mathrm{Ch}}=4 \pi r_{c}{ }^{3} \omega_{R} \rho_{c}=4 \pi \omega_{R}\left[\frac{K}{\pi G_{N}}\right]^{\frac{3}{2}} .
$$

\section{Eddington standard model}

Here, we briefly derive the Eddington standard model which is used in Sec. VA. This model makes several simplifying assumptions in order to model main-sequence stars analytically. The pressure support is assumed to be 
due to two different processes: the motion of the gas, assumed to be ideal

$$
P_{\mathrm{gas}}=\frac{k_{\mathrm{B}} \rho T}{\mu m_{\mathrm{H}}},
$$

where $\mu$ is the mean molecular mass, and radiation pressure,

$$
P_{\mathrm{rad}}=\frac{1}{3} a T^{4} .
$$

The relative importance of each contribution is quantified by

$$
\beta \equiv \frac{P_{\mathrm{gas}}}{P} .
$$

Using Eqs. (A10) and (A11), one finds

$$
\frac{T^{3}}{\rho}=3 a \frac{k_{\mathrm{B}}}{\mu m_{\mathrm{H}}} \frac{1-\beta}{\beta} .
$$

The quantity $T^{3} / \rho$ is the specific entropy density. The key assumption of the Eddington standard model is that this is constant throughout the star. Equation (A13) then implies that $\beta$ is constant. With this assumption, the total pressure is

$$
P=P_{\mathrm{rad}}+P_{\mathrm{gas}}=K(\beta) \rho^{\frac{4}{3}},
$$

with

$$
K(\beta)=\left(\frac{3}{a}\right)^{\frac{1}{3}}\left(\frac{k_{\mathrm{B}}}{\mu m_{\mathrm{H}}}\right)^{\frac{4}{3}}\left(\frac{1-\beta}{\beta^{4}}\right)^{\frac{1}{3}}
$$

We can eliminate $K$ in favor of the mass using Eq. (A7), to find

$$
\frac{1-\beta}{\beta^{4}}=\left(\frac{M}{M_{\mathrm{Edd}}}\right)^{2}
$$

where

$$
M_{\mathrm{Edd}}=\frac{4 \omega_{R}}{\sqrt{\pi} G_{\mathrm{N}}^{\frac{3}{2}}}\left(\frac{3}{a}\right)^{\frac{1}{2}}\left(\frac{k_{\mathrm{B}}}{\mu m_{\mathrm{H}}}\right)^{2}
$$

is the Eddington mass. Equation (A16) is known as Eddington's quartic equation. Its solution gives $\beta(M)$. The key quantity we are interested in is the stellar luminosity, which can be found by inserting $P=(1-\beta(M)) P_{\text {rad }}$ into the hydrostatic equilibrium equation (A2) and substituting this into the equation of radiative transfer,

$$
\frac{\mathrm{d} T}{\mathrm{~d} r}=-\frac{3}{4 a} \frac{\kappa(\rho, T)}{T^{3}} \frac{\rho L}{4 \pi r^{2}}
$$

where $\kappa(\rho, T)$ is the opacity. Making the further assumption that $\kappa$ is constant, one finds

$$
L(M)=\frac{4 \pi(1-\beta(M)) G_{\mathrm{N}} M}{\kappa} .
$$

This relates the luminosity to the mass and $G_{\mathrm{N}}$ and is the equation we use in Sec. VA.
[1] T. Clifton, P. G. Ferreira, A. Padilla, and C. Skordis, Phys. Rep. 513, 1 (2012).

[2] A. Joyce, B. Jain, J. Khoury, and M. Trodden, Phys. Rep. 568, 1 (2015).

[3] K. Koyama, Rep. Prog. Phys. 79, 046902 (2016).

[4] J. Sakstein, Astrophysical Tests of Modified Gravity, Ph.D. thesis, Cambridge University, department of applied mathematics and theoretical physics, 2014.

[5] C. Burrage and J. Sakstein, J. Cosmol. Astropart. Phys. 11 (2016) 045.

[6] C. Burrage and J. Sakstein, Living Rev. Relativity 21, 1 (2018).

[7] J. Sakstein, Int. J. Mod. Phys. D 27, 1848008 (2018).

[8] M. Ishak, Living Rev. Relativity 22, 1 (2019).

[9] J. Khoury, J. Sakstein, and A. R. Solomon, J. Cosmol. Astropart. Phys. 08 (2018) 024.

[10] J. J. Heckman, C. Lawrie, L. Lin, J. Sakstein, and G. Zoccarato, Fortschr. Phys. 67, 1900071 (2019).
[11] A. De Felice, L. Heisenberg, R. Kase, S. Tsujikawa, Y.-1. Zhang, and G.-B. Zhao, Phys. Rev. D 93, 104016 (2016).

[12] C. de Rham, J. T. Deskins, A. J. Tolley, and S.-Y. Zhou, Rev. Mod. Phys. 89, 025004 (2017).

[13] J. Khoury and A. Weltman, Phys. Rev. Lett. 93, 171104 (2004).

[14] J. Khoury and A. Weltman, Phys. Rev. D 69, 044026 (2004).

[15] K. Hinterbichler and J. Khoury, Phys. Rev. Lett. 104, 231301 (2010).

[16] P. Brax, C. van de Bruck, A.-C. Davis, and D. Shaw, Phys. Rev. D 82, 063519 (2010).

[17] A. Vainshtein, Phys. Lett. 39B, 393 (1972).

[18] E. Babichev, C. Deffayet, and R. Ziour, Int. J. Mod. Phys. D 18, 2147 (2009).

[19] J. Sakstein and B. Jain, Phys. Rev. Lett. 119, 251303 (2017). 
[20] P. Creminelli and F. Vernizzi, Phys. Rev. Lett. 119, 251302 (2017).

[21] T. Baker, E. Bellini, P. G. Ferreira, M. Lagos, J. Noller, and I. Sawicki, Phys. Rev. Lett. 119, 251301 (2017).

[22] J. M. Ezquiaga and M. Zumalacárregui, Phys. Rev. Lett. 119, 251304 (2017).

[23] J. Wang, L. Hui, and J. Khoury, Phys. Rev. Lett. 109, 241301 (2012).

[24] J. Sakstein, J. Cosmol. Astropart. Phys. 12 (2014) 012.

[25] H. Y. Ip, J. Sakstein, and F. Schmidt, J. Cosmol. Astropart. Phys. 10 (2015) 051.

[26] E. G. Adelberger, B. R. Heckel, and A. E. Nelson, Annu. Rev. Nucl. Part. Sci. 53, 77 (2003).

[27] E. G. Adelberger, B. R. Heckel, S. A. Hoedl, C. D. Hoyle, D. J. Kapner, and A. Upadhye, Phys. Rev. Lett. 98, 131104 (2007).

[28] E. G. Adelberger, J. H. Gundlach, B. R. Heckel, S. Hoedl, and S. Schlamminger, Prog. Part. Nucl. Phys. 62, 102 (2009).

[29] J. Sakstein, Phys. Rev. D 97, 064028 (2018).

[30] L. Berezhiani, J. Khoury, and J. Wang, Phys. Rev. D 95, 123530 (2017).

[31] D. Bettoni and S. Liberati, Phys. Rev. D 88, 084020 (2013).

[32] M. Zumalacárregui and J. García-Bellido, Phys. Rev. D 89, 064046 (2014).

[33] J. Gleyzes, D. Langlois, F. Piazza, and F. Vernizzi, Phys. Rev. Lett. 114, 211101 (2015).

[34] D. Bettoni and M. Zumalacárregui, Phys. Rev. D 91, 104009 (2015).

[35] C. M. Will, Pramana 63, 731 (2004).

[36] A.-C. Davis, E. A. Lim, J. Sakstein, and D. Shaw, Phys. Rev. D 85, 123006 (2012).

[37] B. Jain, V. Vikram, and J. Sakstein, Astrophys. J. 779, 39 (2013).

[38] V. Vikram, J. Sakstein, C. Davis, and A. Neil, Phys. Rev. D 97, 104055 (2018).

[39] K. Koyama and J. Sakstein, Phys. Rev. D 91, 124066 (2015).

[40] J. Sakstein, Phys. Rev. Lett. 115, 201101 (2015).

[41] J. Sakstein, Phys. Rev. D 92, 124045 (2015).

[42] J. Sakstein, M. Kenna-Allison, and K. Koyama, J. Cosmol. Astropart. Phys. 03 (2017) 007.

[43] E. Babichev, K. Koyama, D. Langlois, R. Saito, and J. Sakstein, Classical Quantum Gravity 33, 235014 (2016).

[44] J. Sakstein, E. Babichev, K. Koyama, D. Langlois, and R. Saito, Phys. Rev. D 95, 064013 (2017).

[45] J. Sakstein, H. Wilcox, D. Bacon, K. Koyama, and R. C. Nichol, J. Cosmol. Astropart. Phys. 07 (2016) 019.

[46] J. Sakstein, B. Jain, J. S. Heyl, and L. Hui, Astrophys. J. 844, L14 (2017).

[47] S. Adhikari, J. Sakstein, B. Jain, N. Dalal, and B. Li, J. Cosmol. Astropart. Phys. 11 (2018) 033.

[48] H. Desmond, P. G. Ferreira, G. Lavaux, and J. Jasche, Phys. Rev. D 98, 064015 (2018).

[49] H. Desmond, P. G. Ferreira, G. Lavaux, and J. Jasche, Mon. Not. R. Astron. Soc. 483, L64 (2019).

[50] H. Desmond, P. G. Ferreira, G. Lavaux, and J. Jasche, Phys. Rev. D 98, 083010 (2018).
[51] S. Dubovsky, T. Gregoire, A. Nicolis, and R. Rattazzi, J. High Energy Phys. 03 (2006) 025.

[52] B. Bertotti, L. Iess, and P. Tortora, Nature (London) 425, 374 (2003).

[53] S. Weinberg, Phys. Rev. 140, B516 (1965).

[54] J. I. Read, J. Phys. G 41, 063101 (2014).

[55] J. Buch, S. C. J. Leung, and J. Fan, J. Cosmol. Astropart. Phys. 04 (2019) 026.

[56] J. P. Cox, Research Supported by the National Science Foundation Princeton (Princeton University, Princeton, NJ, 1980), p. 393.

[57] H. Y. Xu and Y. Li, Astron. Astrophys. 418, 213 (2004).

[58] J. J. Walmswell, C. A. Tout, and J. J. Eldridge, Mon. Not. R. Astron. Soc. 447, 2951 (2015).

[59] A. Schootemeijer, N. Langer, N. J. Grin, and C. Wang, Astron. Astrophys. 625, A132 (2019).

[60] R. Kippenhahn and A. Weigert, Stellar Structure and Evolution (Springer-Verlag, Berlin, 1990; Also Astronomy and Astrophysics Library), p. 192.

[61] B. Paxton, P. Marchant, J. Schwab, E. B. Bauer, L. Bildsten, M. Cantiello, L. Dessart, R. Farmer, H. Hu, N. Langer, R. H. D. Townsend, D. M. Townsley, and F. X. Timmes, Astrophys. J. Suppl. Ser. 220, 15 (2015).

[62] B. Paxton et al., Astrophys. J. Suppl. Ser. 234, 34 (2018).

[63] B. Paxton, R. Smolec, A. Gautschy, L. Bildsten, M. Cantiello, A. Dotter, R. Farmer, J. A. Goldberg, A. S. Jermyn, S. M. Kanbur, P. Marchant, J. Schwab, A. Thoul, R. H. D. Townsend, W. M. Wolf, M. Zhang, and F. X. Timmes, Astron. Astrophys. Suppl. Ser. 243, 10 (2019).

[64] M. F. El Eid, Mon. Not. R. Astron. Soc. 275, 983 (1995).

[65] W. L. Freedman and B. F. Madore, Annu. Rev. Astron. Astrophys. 48, 673 (2010).

[66] M. R. Becker, H. Desmond, E. Rozo, P. Marshall, and E. S. Rykoff, arXiv:1507.07523.

[67] J. Sakstein, Phys. Rev. D 88, 124013 (2013).

[68] B. Paxton, L. Bildsten, A. Dotter, F. Herwig, P. Lesaffre, and F. Timmes, Astrophys. J. Suppl. Ser. 192, 3 (2011).

[69] F. Herwig, Astron. Astrophys. 360, 952 (2000).

[70] I. P. Lopes and J. Silk, Mon. Not. R. Astron. Soc. 341, 721 (2003).

[71] F. C. Adams, J. Cosmol. Astropart. Phys. 08 (2008) 010.

[72] H. Desmond, B. Jain, and J. Sakstein, Phys. Rev. D 100, 043537 (2019).

[73] B. S. Wright and B. Li, Phys. Rev. D 97, 083505 (2018).

[74] M. Crisostomi, M. Lewandowski, and F. Vernizzi, Phys. Rev. D 100, 024025 (2019).

[75] T. E. Collett, L. J. Oldham, R. J. Smith, M. W. Auger, K. B. Westfall, D. Bacon, R. C. Nichol, K. L. Masters, K. Koyama, and R. van den Bosch, Science 360, 1342 (2018).

[76] J. Schwab, A. S. Bolton, and S. A. Rappaport, Astrophys. J. 708, 750 (2010).

[77] A. Terukina, L. Lombriser, K. Yamamoto, D. Bacon, K. Koyama, and R. C. Nichol, J. Cosmol. Astropart. Phys. 04 (2014) 013.

[78] H. Wilcox et al., Mon. Not. R. Astron. Soc. 452, 1171 (2015).

[79] L. Pizzuti et al., J. Cosmol. Astropart. Phys. 04 (2016) 023.

[80] F. Simpson et al., Mon. Not. R. Astron. Soc. 429, 2249 (2013). 
[81] Y.-S. Song, G.-B. Zhao, D. Bacon, K. Koyama, R. C. Nichol, and L. Pogosian, Phys. Rev. D 84, 083523 (2011).

[82] C. Blake et al., Mon. Not. R. Astron. Soc. 456, 2806 (2016).

[83] S. Singh, S. Alam, R. Mandelbaum, U. Seljak, S. Rodriguez-Torres, and S. Ho, Mon. Not. R. Astron. Soc. 482, 785 (2019).

[84] S. Hirano, T. Kobayashi, and D. Yamauchi, Phys. Rev. D 99, 104073 (2019).

[85] B. Abbott et al. (LIGO Scientific, Virgo Collaborations), Phys. Rev. Lett. 119, 161101 (2017).

[86] M. Crisostomi and K. Koyama, Phys. Rev. D 97, 084004 (2018).

[87] E. J. Copeland, M. Kopp, A. Padilla, P. M. Saffin, and C. Skordis, Phys. Rev. Lett. 122, 061301 (2019).

[88] R. Kase and S. Tsujikawa, Int. J. Mod. Phys. D 28, 1942005 (2019).

[89] T. Kobayashi, Rep. Prog. Phys. 82, 086901 (2019).

[90] C. de Rham and S. Melville, Phys. Rev. Lett. 121, 221101 (2018).

[91] J. Beltran Jimenez, F. Piazza, and H. Velten, Phys. Rev. Lett. 116, 061101 (2016).
[92] A. G. Riess, S. Casertano, W. Yuan, L. M. Macri, and D. Scolnic, Astrophys. J. 876, 85 (2019).

[93] R. S. Goncalves, G. C. Carvalho, and J. S. Alcaniz, Phys. Rev. D 92, 123504 (2015).

[94] A. Barreira, B. Li, C. M. Baugh, and S. Pascoli, Phys. Rev. D 86, 124016 (2012).

[95] J. Renk, M. Zumalacárregui, F. Montanari, and A. Barreira, J. Cosmol. Astropart. Phys. 10 (2017) 020.

[96] B. Jain and J. VanderPlas, J. Cosmol. Astropart. Phys. 10 (2011) 032.

[97] V. Vikram, A. Cabré, B. Jain, and J. T. VanderPlas, J. Cosmol. Astropart. Phys. 08 (2013) 020.

[98] T. Hiramatsu, W. Hu, K. Koyama, and F. Schmidt, Phys. Rev. D 87, 063525 (2013).

[99] J. Sakstein, Phys. Rev. D 91, 024036 (2015).

[100] J. Sakstein and S. Verner, Phys. Rev. D 92, 123005 (2015).

[101] P. Brax and A.-C. Davis, Phys. Rev. D 98, 063531 (2018).

[102] A. Kuntz, F. Piazza, and F. Vernizzi, J. Cosmol. Astropart. Phys. 05 (2019) 052.

[103] P. Brax, A.-C. Davis, and A. Kuntz, Phys. Rev. D 99, 124034 (2019). 